\title{
TNFR2 limits proinflammatory astrocyte functions during EAE induced by pathogenic DR2b-restricted T cells
}

\author{
Itay Raphael,, ${ }^{1,2}$ Francisco Gomez-Rivera, ${ }^{2,3}$ Rebecca A. Raphael,, ${ }^{2,4}$ Rachel R. Robinson, ${ }^{2}$ \\ Saisha Nalawade, ${ }^{2,5}$ and Thomas G. Forsthuber ${ }^{2}$ \\ 'Department of Neurological Surgery, University of Pittsburgh, UPMC Children's Hospital, Pittsburgh, Pennsylvania, USA. \\ ${ }^{2}$ Department of Biology, University of Texas at San Antonio, San Antonio, Texas, USA. ${ }^{3}$ Department of Internal Medicine, \\ University of Michigan, Ann Arbor, Michigan, USA. ${ }^{4}$ Department of Pharmacology and Chemical Biology, University of \\ Pittsburgh, UPMC Hillman Cancer Center, Pittsburgh, Pennsylvania, USA. ${ }^{5}$ Center for Cell and Gene Therapy, Baylor College \\ of Medicine, Houston, Texas, USA.
}

Multiple sclerosis (MS) is an autoimmune neuroinflammatory disease where the underlying mechanisms driving disease progression have remained unresolved. HLA-DR2b (DRB1*15:01) is the most common genetic risk factor for MS. Additionally, TNF and its receptors TNFR1 and TNFR2 play key roles in MS and its preclinical animal model, experimental autoimmune encephalomyelitis (EAE). TNFR2 is believed to ameliorate CNS pathology by promoting remyelination and Treg function. Here, we show that transgenic mice expressing the human MHC class II (MHC-II) allele HLA-DR2b and lacking mouse MHC-II and TNFR2 molecules, herein called DR2b $\Delta R 2$, developed progressive EAE, while disease was not progressive in DR2b littermates. Mechanistically, expression of the HLA-DR2b favored Th17 cell development, whereas T cell-independent TNFR2 expression was critical for restraining of an astrogliosis-induced proinflammatory milieu and Th17 cell responses, while promoting remyelination. Our data suggest the TNFR2 signaling pathway as a potentially novel mechanism for curtailing astrogliosis and promoting remyelination, thus providing new insights into mechanisms limiting progressive MS.

Conflict of interest: The authors have declared that no conflict of interest exists.

Copyright: (c) 2019, American Society for Clinical Investigation.

Submitted: August 9, 2019

Accepted: November 13, 2019

Published: December 19, 2019

Reference information: /CI Insight. 2019;4(24):e132527.

https://doi.org/10.1172/jci.

insight.132527.

\section{Introduction}

Multiple sclerosis (MS) is an autoimmune neuroinflammatory disease of the CNS that affects over 400,000 Americans and approximately 2.5 million people worldwide (1). It is mediated by neuroantigen-reactive $\mathrm{T}$ cells that mount an immune attack against the myelin sheath of neurons, leading to demyelination, axonal loss, and subsequently neurological impairments and other clinical symptoms (2). Approximately $85 \%$ of patients are diagnosed with relapsing-remitting MS at onset. However, about half of these patients develop secondary-progressive MS by 10-20 years after primary diagnosis. Moreover, approximately $15 \%$ of MS patients are diagnosed with progressive MS from disease onset $(1,3)$. The underlying mechanisms that promote MS disease progression and exacerbation remain elusive, and therefore there are currently no treatments available to prevent or reverse this process; thus progressive MS remains one of the most pressing clinical problems (3).

MS susceptibility is thought to be mediated by both genetic and environmental factors. The MHC class II (MHC-II) gene, HLA-DR2b (DRB1*15:01), is the most common genetic link to MS, although the mechanisms by which it may promote disease progression are not fully understood (4). The TCR-MHC complex plays a key role in thymic $\mathrm{T}$ cell development and in guiding $\mathrm{T}$ cell subset differentiation and effector responses upon antigen $(\mathrm{Ag})$ encounter $(5,6)$. Therefore, autoimmune disease-associated HLA molecules, such as HLA-DR2b, may contribute to disease via the formation of autoreactive, pathogenic $\mathrm{T}$ cells and by shaping $\mathrm{T}$ cell tolerance and the Treg compartment.

TNF is a pleiotropic cytokine with important physiological and pathogenic functions, for example in promoting disease in MS, rheumatoid arthritis, and inflammatory bowel disease (7). In patients with MS, TNF expression in cerebrospinal fluid correlates with disease progression, which suggests that TNF plays a key role in progression of MS (8). Likewise, studies in experimental autoimmune encephalomyelitis 
A

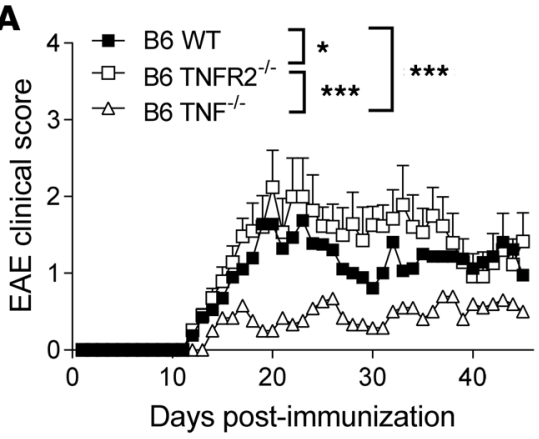

C Spleen (naive)

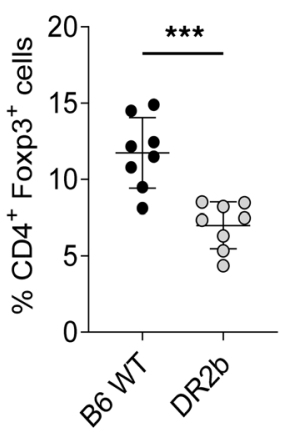

$\mathbf{F}$

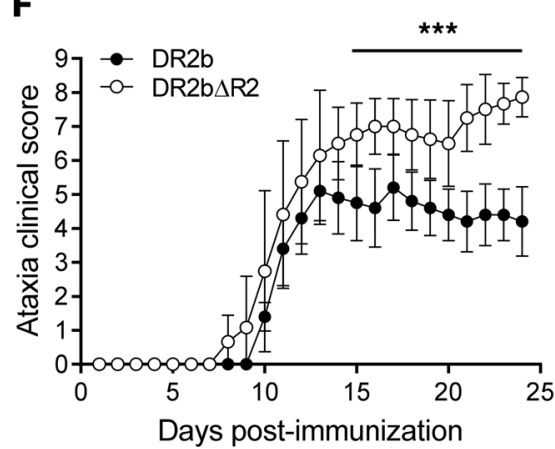

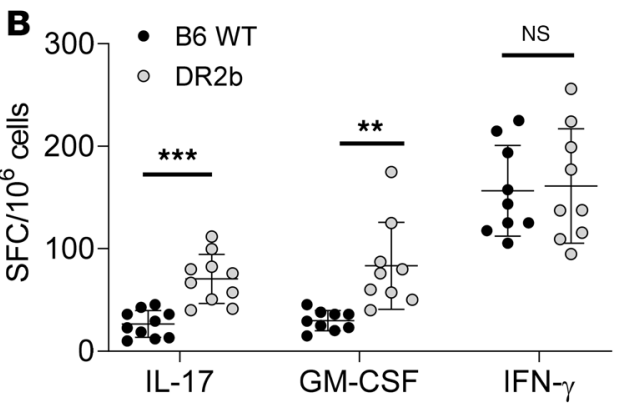

E

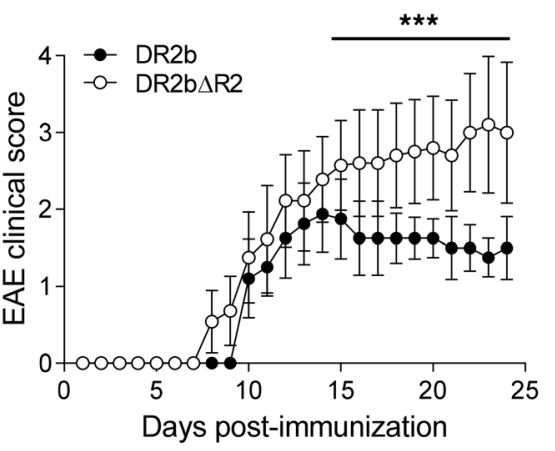

G

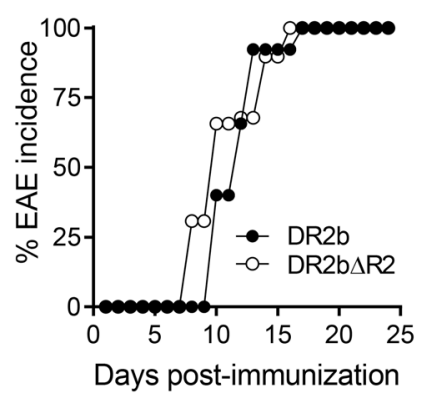

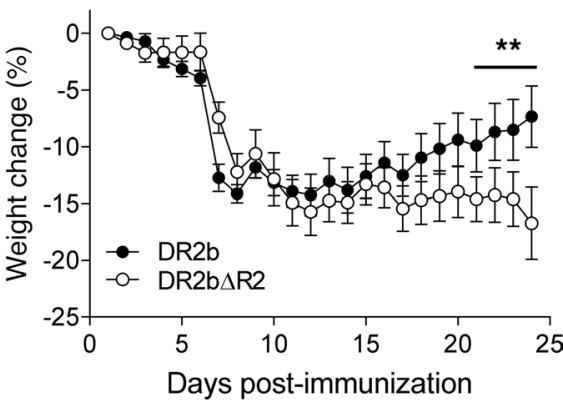

H

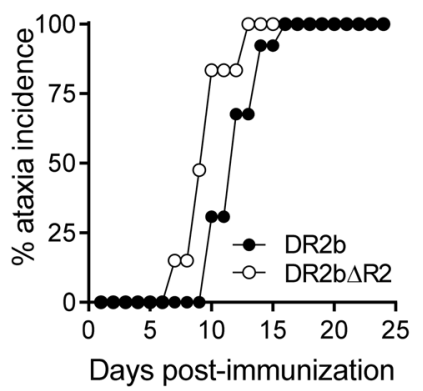

Figure 1. Lack of TNFR2 expression promotes the development of progressive experimental autoimmune encephalomyelitis in HLA-DR2b-transgenic mice. (A) C57BL/6 (B6) wild-type (WT), B6 TNFR2 ${ }^{-1-}$, and B6 $\mathrm{TNF}^{-1-}$ mice were immunized to induce experimental autoimmune encephalomyelitis (EAE). Clinical signs of EAE were monitored daily. Shown are mean clinical disease scores of representative results from 3-6 independent experiments with $n=5-12$ mice per group. Statistical significance was determined by 1-way ANOVA corrected for FDR using Benjamini, Krieger, and Yekutieli method. (B) B6 WT and DR2b-transgenic mice were immunized with $\mathrm{MOG}_{35-55}$ peptide in CFA, and the frequencies of Ag-reactive IL-17-, GM-CSF-, and IFN- $\gamma$-producing T cells were measured in lymph nodes and spleens at day 9 after immunization. Pooled data from 3 independent experiments, $n=9-10$ mice per group. (C) Percentage of CD4 ${ }^{+}$Foxp $3^{+}$cells in naive WT B6 mice and naive DR2b mice. Pooled data from 2 independent experiments, $n=8$ mice per group. Student's 2-tailed $t$ test with Welch's correction. (D-H) DR2b $\left(\mathrm{DR} 2 \mathrm{~b}^{+/+} \mathrm{I}-\mathrm{A}^{-/-}\right.$) and DR2b $\triangle \mathrm{R} 2\left(\mathrm{DR} 2 \mathrm{~b}^{+/+} \mathrm{I}-\mathrm{A}^{-/-}\right.$TNFR2 ${ }^{-/-}$) were immunized to induce EAE. Shown are representative results from 3-6 independent experiments with $n=5-10$ mice per group. (D) Clinical signs of EAE, (E) clinical signs of weight loss, and (F) clinical signs of ataxia were monitored daily. (C) EAE disease incidence and $(\mathbf{H})$ clinical ataxia incidence were evaluated daily. Statistical significance was determined by multiple comparisons with Holm-Šídák correction (B, D-F). NS, not significant; ${ }^{*} P \leq 0.05 ;{ }^{* *} P \leq 0.01$; and ${ }^{* * *} P \leq 0.001$. Error bars indicate mean \pm standard deviation (SD).

(EAE), the preclinical animal model of MS, revealed key pathogenic functions for TNF signaling. Thus, targeting TNF was suggested as an attractive therapy for MS. Surprisingly, however, TNF-blocking drugs with proven efficacy in other autoimmune diseases triggered onset and exacerbations of MS, which provided evidence for a role of TNF in promoting CNS protection and repair $(9,10)$.

TNF exerts its function via binding of TNF receptor (TNFR) 1 (TNFRSF1A) or TNFR2 (TNFRSF1B), which have nonredundant roles. Soluble and membrane-bound TNF can bind to both receptors, though membrane-bound TNF is a more potent ligand for TNFR2. Most cells constitutively express low levels of TNFR1, while the expression of TNFR2 is restricted mostly to immune and endothelial cells. Mice lacking TNF or TNFR1 are resistant to EAE development, while clinical disease is more severe in 
TNFR2 $^{-/-}$mice $(11,12)$. The exacerbated disease observed in TNFR2-deficient mice is primarily attributed to defects in the Treg cell compartment, increased effector function of inflammatory monocytes, and impaired remyelination (13-16). Nevertheless, inflammation can trigger the expression of TNFR2 in glial cells and promote protective CNS functions, including endowing microglia with antiinflammatory properties and promoting oligodendrocyte proliferation and remyelination (14, 17-19).

TNFR2 expression in astrocytes was suggested to promote remyelination by inducing the expression of cytokines, which promote oligodendrocyte precursor cell (OPC) proliferation and maturation in areas of demyelination $(16,20)$. Importantly, genome-wide association studies revealed a genetic link between a single nucleotide polymorphism in TNFR1 (rs4149584) and MS but not other autoimmune diseases (21, 22). Functional studies of the TNFR1 rs4149584 variant showed that it directs expression of a soluble form of TNFR1 capable of binding to and inhibiting TNF signaling, thereby mimicking the effect of TNF-blocking drugs in MS $(23,24)$. Together these data support a concept in which TNFR1 is pathogenic in MS and EAE, while TNFR2 is protective.

Here, we hypothesized that T cell-independent expression of TNFR2 modulates the encephalitogenic function of neuroantigen-reactive $\mathrm{T}$ cells generated in the context of the MS-associated HLA-DR2b allele. To address this question, we generated a mouse model, HLA-DR2 $\mathrm{b}^{+/+} \mathrm{I}_{-} \mathrm{A}^{-/-} \mathrm{TNFR}^{-/}$, herein called $\mathrm{DR} 2 \mathrm{~b} \Delta \mathrm{R} 2$, which expresses human MHC-II HLA-DR2b molecules, but not endogenous murine MHC-II molecules $\left(\mathrm{I}-\mathrm{A}^{\mathrm{b}}\right)$, and lacks expression of TNFR2. DR2b $\Delta \mathrm{R} 2$ mice developed progressive EAE, while DR2b littermate controls, or TNFR2 ${ }^{-1-}$ mice on a C57BL/ 6 background expressing I-A ${ }^{\mathrm{b}}$, did not. Furthermore, we showed that lack of TNFR2 signaling by CNS-resident cells resulted in decreased OPC-dependent remyelination, pronounced astrogliosis, and enhanced proinflammatory functions. Our work provides key insights for future generation of novel treatments and understanding of progressive MS.

\section{Results}

TNFR2 signaling restrains the development of progressive EAE in HLA-DR2b-transgenic mice. Previous studies have implicated TNF and its receptor TNFR1 in the pathogenesis of MS and EAE $(11,25,26)$. Specifically, TNF signaling via TNFR1 promotes pathogenicity in EAE, while TNFR2 is critical for neuroprotection (27). In agreement with these studies, $\mathrm{B} 6 \mathrm{TNF}^{-/-}$mice were resistant to $\mathrm{EAE}$, whereas $\mathrm{B} 6 \mathrm{TNFR} 2^{-/-}$mice were fully susceptible to disease and exhibited more severe clinical signs compared with EAE-susceptible B6 WT mice (Figure 1A). Furthermore, both B6 TNFR2 ${ }^{-/-}$mice and B6 WT mice exhibited clinical remission (Figure 1A).

The MHC-II allele HLA-DR2b (DRB1*15:01) is associated with MS susceptibility and EAE development (28). Therefore, we analyzed $\mathrm{T}$ cell responses in HLA-DR2b-transgenic (HLA-DR2b-Tg) mice lacking endogenous murine I-A ${ }^{b}$ MHC-II molecules (herein referred to as DR2b mice) and B6 WT mice after immunization with $\mathrm{MOG}_{35-55}$ peptide. Of note, DR2b mice generated robust $\mathrm{MOG}_{35-55}$-specific IL-17- and GM-CSF-producing T cell responses with significantly higher frequencies compared with I-A ${ }^{b}$-restricted B6 WT mice (Figure 1B). However, we did not observe significant differences in the frequencies of $\mathrm{MOG}_{35-55}$-specific IFN- $\gamma$-producing Th1 cells (Figure 1B). Furthermore, naive DR2b mice showed lower percentages of Foxp $3^{+}$Treg cells than B6 WT animals (Figure 1C), in accordance with previous results (29). Thus, the results suggested that the expression of human DR2b favors the generation of pathogenic T cells while impairing Treg cell development.

Next, we investigated the role that TNFR2 plays in modulating the function of HLA-DR2b-restricted

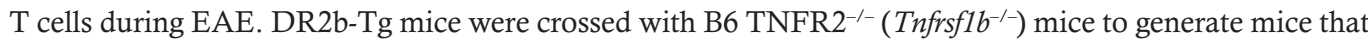
expressed the human DR2b MHC-II allele in the absence of TNFR2, hereinafter designated DR2b $\Delta$ R2 mice (Supplemental Figure 1A; supplemental material available online with this article; https://doi. org/10.1172/jci.insight.132527DS1). We confirmed the presence of DR2b and the absence of TNFR2 and murine I-A ${ }^{\mathrm{b}} \mathrm{MHC}-\mathrm{II}$ molecules in DR2b $\Delta \mathrm{R} 2$ mice (Supplemental Figure 1, B-E).

Notably, DR2b $\Delta$ R2 mice consistently showed increased EAE disease severity compared with DR2b littermates (Figure 1D). Importantly, DR2b $\Delta \mathrm{R} 2$ mice developed progressive EAE and did not show clinical remission (Figure 1, D-F), whereas disease in DR2b mice (Figure 1, D-F) and B6 TNFR2 ${ }^{-/}$mice (Figure 1A) was not progressive and the animals exhibited clinical remission. Interestingly, DR2b $\Delta R 2$ mice developed cerebellar ataxia, which was significantly more pronounced than that of DR2b mice and from which they consistently did not recover (Figure $1 F$ ). Additionally, onset of EAE and ataxia in DR2b $\Delta$ R2 animals was somewhat earlier, though disease incidence at the peak of disease was comparable (Figure $1, \mathrm{G}$ and $\mathrm{H}$ ). 
A

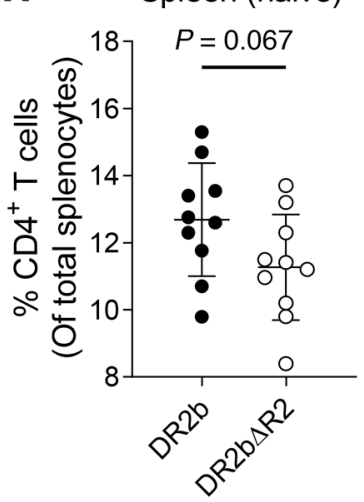

B Spleen (naive)

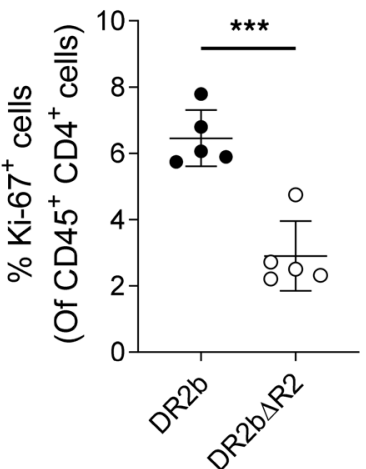

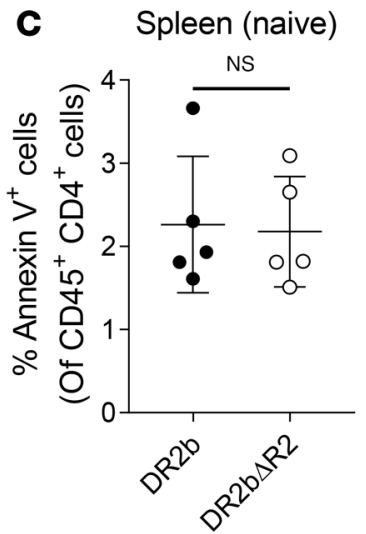

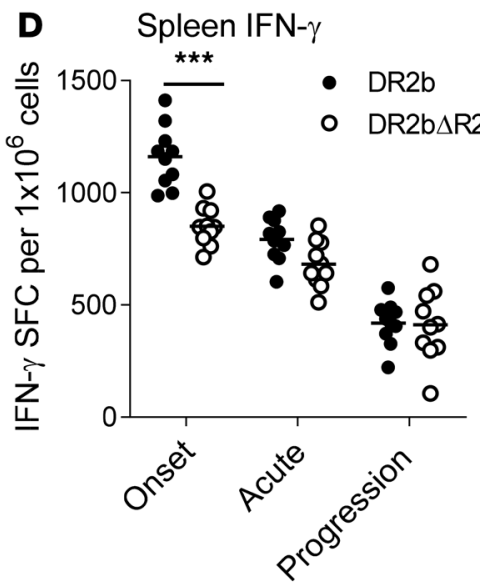

E

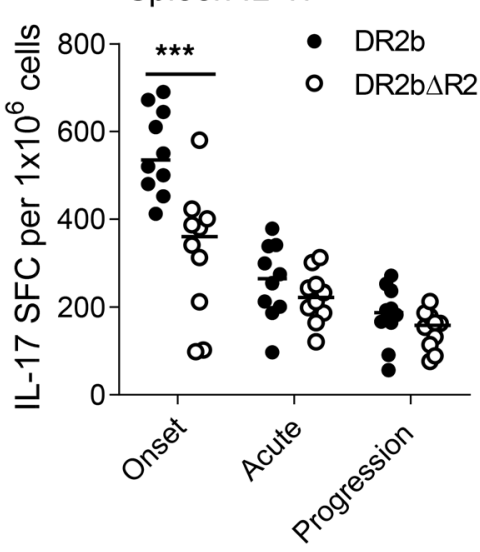

F

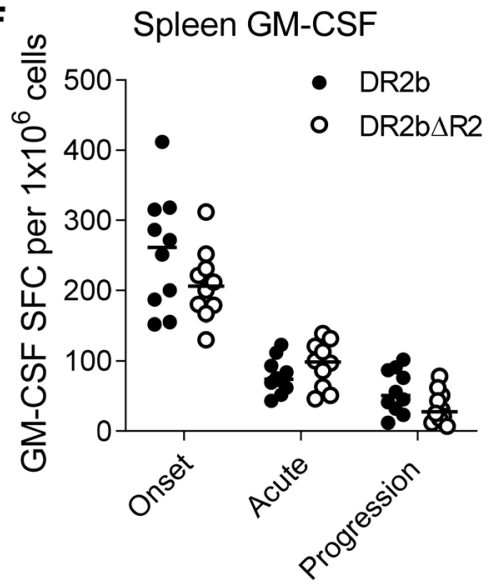

G Spleen

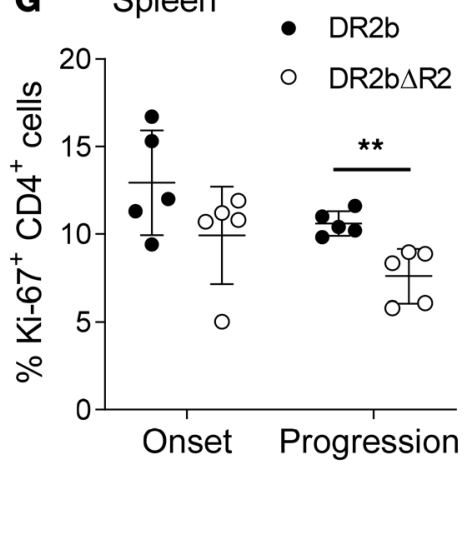

\section{H Spleen (progression)}

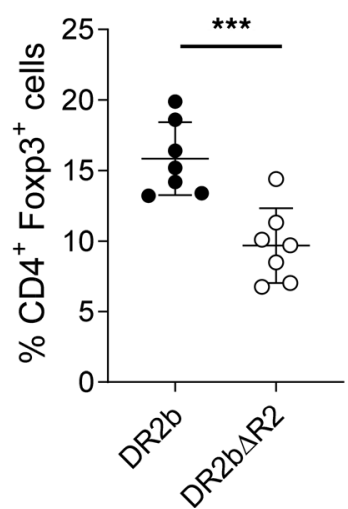

I Spleen (naive)

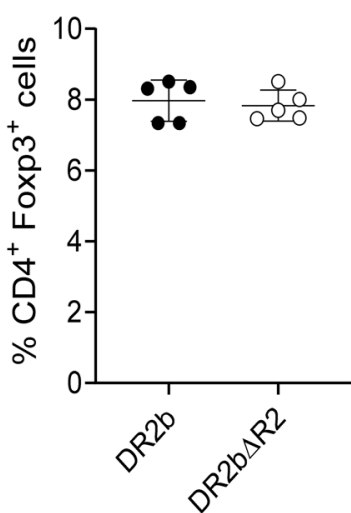

J Serum (progression)

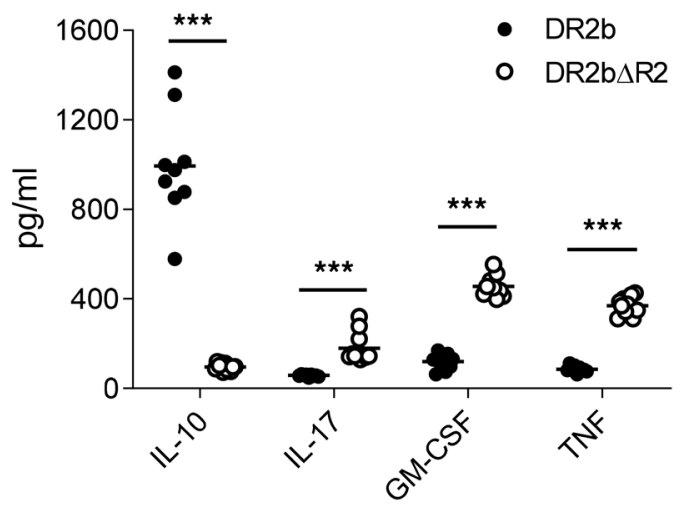

Figure 2. TNFR2 signaling modulates CD4+ $\mathbf{T}$ cell immunity and Treg cells in DR2b mice. (A) Percentage of CD4 ${ }^{+} \mathrm{T}$ cells in splenocytes from naive $\mathrm{DR} 2 \mathrm{~b}$ and DR2b $\triangle$ R2 mice. Pooled data of 3 independent experiments, $n=10$ mice per group. (B) Ki-67 and (C) Annexin V expression in CD4 ${ }^{+}$T cells from spleen of naive DR2b and DR2b $\Delta R 2$ mice. Representative results from 3 (B) and 4 (C) independent experiments with $n=3-5$ per group. (D-F) Frequencies of $\mathrm{MOG}_{35-55}$-specific (D) IFN- $\gamma-$, (E) IL-17-, and (F) GM-CSF-producing T cells in spleens of DR2b and DR2b DR2b $\triangle R 2$ mice immunized for EAE at day 10 (onset), day 15 (acute), and day 24 (progression) after immunization measured by cytokine ELISPOT assay. Representative results from 5 independent experiments, $n=10$ mice per group. Expression of (G) Ki-67 and (H and I) Foxp3 by CD4 ${ }^{+}$T cells isolated from spleens at indicated time points during EAE. Representative results from 3 independent experiments, $n=4-5$ mice per group. (J) Serum concentration of IL-10, IL-17, $\mathrm{CM}-\mathrm{CSF}$, and TNF during the progression phase of EAE in DR2b and DR2b $\triangle R 2$ mice. Pooled data from 2 independent experiments with $n=9$ for DR2b mice and $n=11$ for DR2b $\Delta$ R2 mice. Statistical significance was determined by Student's 2-tailed $t$ test with Holm-Šídák (D-F, G) or Welch's (A-C and $\mathbf{G}-\mathrm{I}$ ) correction. NS, not significant; ${ }^{*} P \leq 0.01$; and ${ }^{* *} P \leq 0.001$. Shown are means. Error bars indicate SD. 

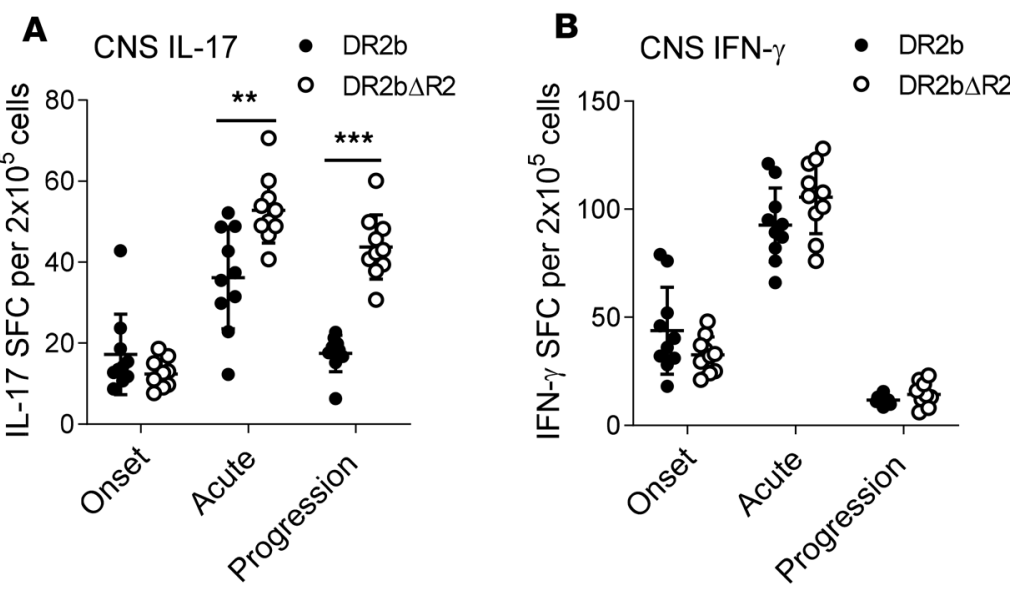

D

CNS Tregs

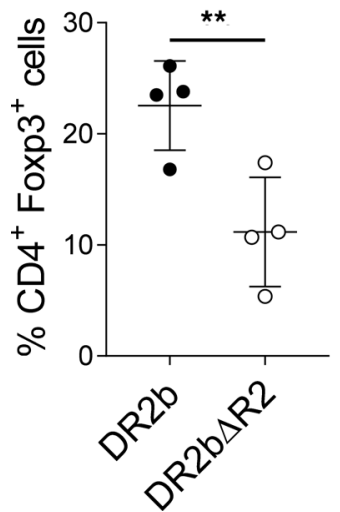

E CNS lesions

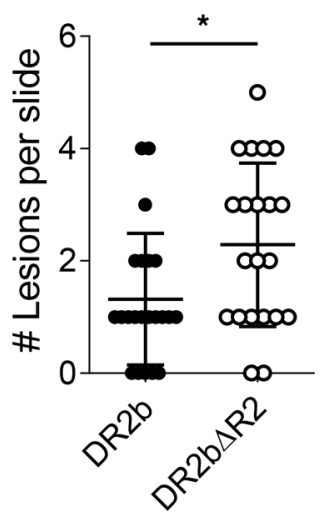

$\mathbf{F}$

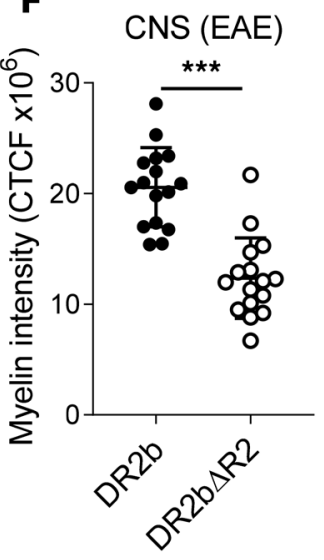

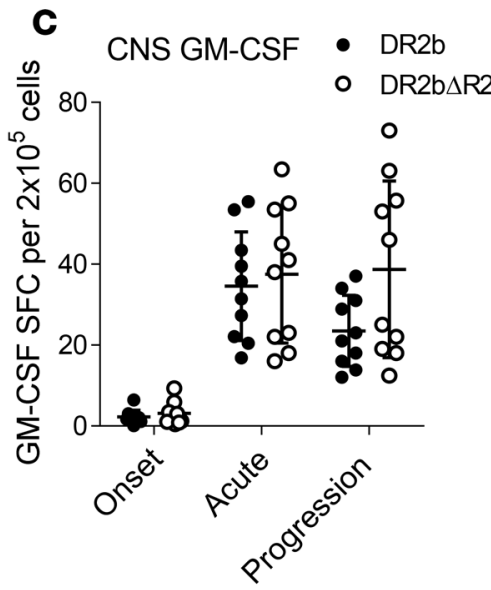

Figure 3. TNFR2 attenuates Th17 cell responses in the CNS of DR2b mice and decreases CNS pathology. (A-C) Frequencies of $M_{0} \mathrm{C}_{35-55}{ }^{-S p e c i f i c ~(A) ~ I L-17-, ~}$ (B) IFN- $\gamma-$, and (C) CM-CSF-producing T cells in CNS tissue of DR2b and DR2b $\triangle R 2$ mice immunized for EAE at onset, acute, and disease progression phases measured by cytokine ELISPOT assay. (D) Frequencies of $\mathrm{CD}^{+}{ }^{+}$Foxp $3^{+}$cells in CNS tissue at acute disease in DR2b and DR2b $\Delta R 2$ mice measured by flow cytometry. (E) Number of lesions per CNS tissue slide from cerebellum and spinal cord sections at acute disease. (F and $\mathbf{G})$ Myelin fluorescence intensity measured by immunofluorescence (IF) microscopy in the cerebellum of (F) EAE mice at the progression phase and in (G) naive animals. CTCF, corrected total cell fluorescence. Representative data from $5(\mathbf{A}-\mathbf{C})$ or $3(\mathbf{D})$ independent experiments with $n=5-10$ mice $(\mathbf{A}-\mathbf{C})$ or $n=3-5$ mice (D) per group. (E and F) Pooled data from 3 independent experiments with $n=4-6$ mice per group. (G) Pooled data from 2 independent experiments with $n=8$ mice per group. Statistical significance was determined by Student's 2-tailed $t$ test with Welch's correction (A-D, $\mathbf{F}$, and $\mathbf{G})$ or Mann-Whitney $U$ test $(U=142.5)(\mathbf{E})$. ${ }^{*} P \leq$ $0.05,{ }^{*} P \leq 0.01$, and ${ }^{* *} P \leq 0.001$. Error bars indicate mean \pm SD.

Taken together, DR2b $\Delta \mathrm{R} 2$ mice developed EAE with progressively increasing disease severity and ataxia without clinical remission. Cerebellar ataxia is a leading feature of MS, in particular of progressive disease, and therefore $\mathrm{EAE}$ in $\mathrm{DR} 2 \mathrm{~b} \triangle \mathrm{R} 2$ mice replicated some of the cardinal features observed in patients (30). Together, the data suggested a role for TNFR2 signaling in restraining progressive EAE mediated by neuroantigen-specific T cells formed in the context of MS-associated HLA-DR2b.

TNFR2 signaling restricts inflammation and maintains Treg cell numbers in naive HLA-DR2b-Tg mice and during $E A E$. TNF plays important roles in apoptosis and survival of $\mathrm{CD}^{+} \mathrm{T}$ lymphocytes; therefore, we investigated the effect of TNFR2 deficiency on $\mathrm{CD}^{+} \mathrm{T}$ cell immunity in naive HLA-DR2b-Tg mice and during EAE.

Investigation of $\mathrm{T}$ cells in the spleen of naive $\mathrm{DR} 2 \mathrm{~b} \Delta \mathrm{R} 2$ mice showed that the percentages of $\mathrm{CD} 4^{+} \mathrm{T}$ cells tended to be lower than in DR2b controls (Figure 2A). Notably, we observed a significant reduction in proliferating $\mathrm{CD}^{+} \mathrm{T}$ cells in naive $\mathrm{DR} 2 \mathrm{~b} \Delta \mathrm{R} 2$ mice (Figure $2 \mathrm{~B}$ ). However, $\mathrm{CD} 4^{+} \mathrm{T}$ cell apoptosis in naive $\mathrm{DR} 2 \mathrm{~b}$ and $\mathrm{DR} 2 \mathrm{~b} \Delta \mathrm{R} 2$ animals was comparable (Figure $2 \mathrm{C}$ ). The data suggest that TNFR2 modulates naive $\mathrm{CD}^{+} \mathrm{T}$ cell proliferation in this model without affecting their survival, consistent with previous findings (31).

Next, we investigated TNFR2-mediated effects on $\mathrm{CD}^{+} \mathrm{T}$ cell effector function during EAE. We consistently detected a modest decrease in the frequencies of $\mathrm{MOG}_{35-55}$-reactive $\mathrm{T}$ cells producing IFN- $\gamma$, IL-17, and GM-CSF at onset (day 10 after immunization) in spleen and lymph nodes of DR2b $\Delta$ R2 mice, 
A

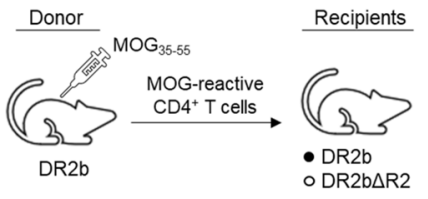

D

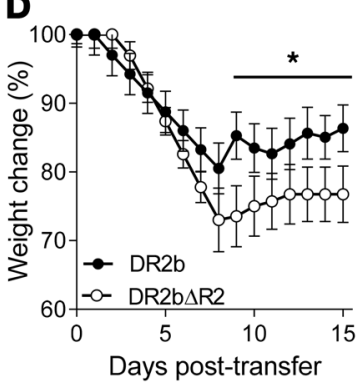

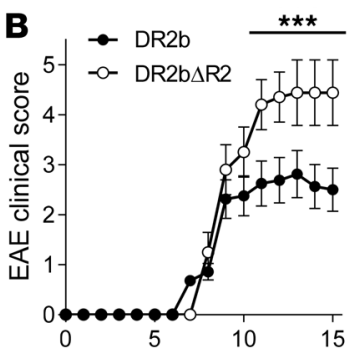

Days post-transfer

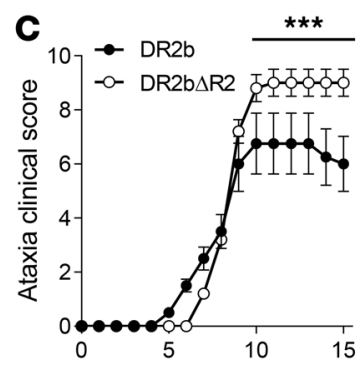

Days post-transfer

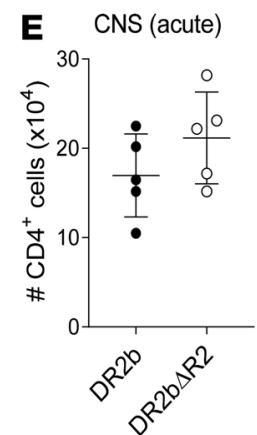

F CNS (acute)
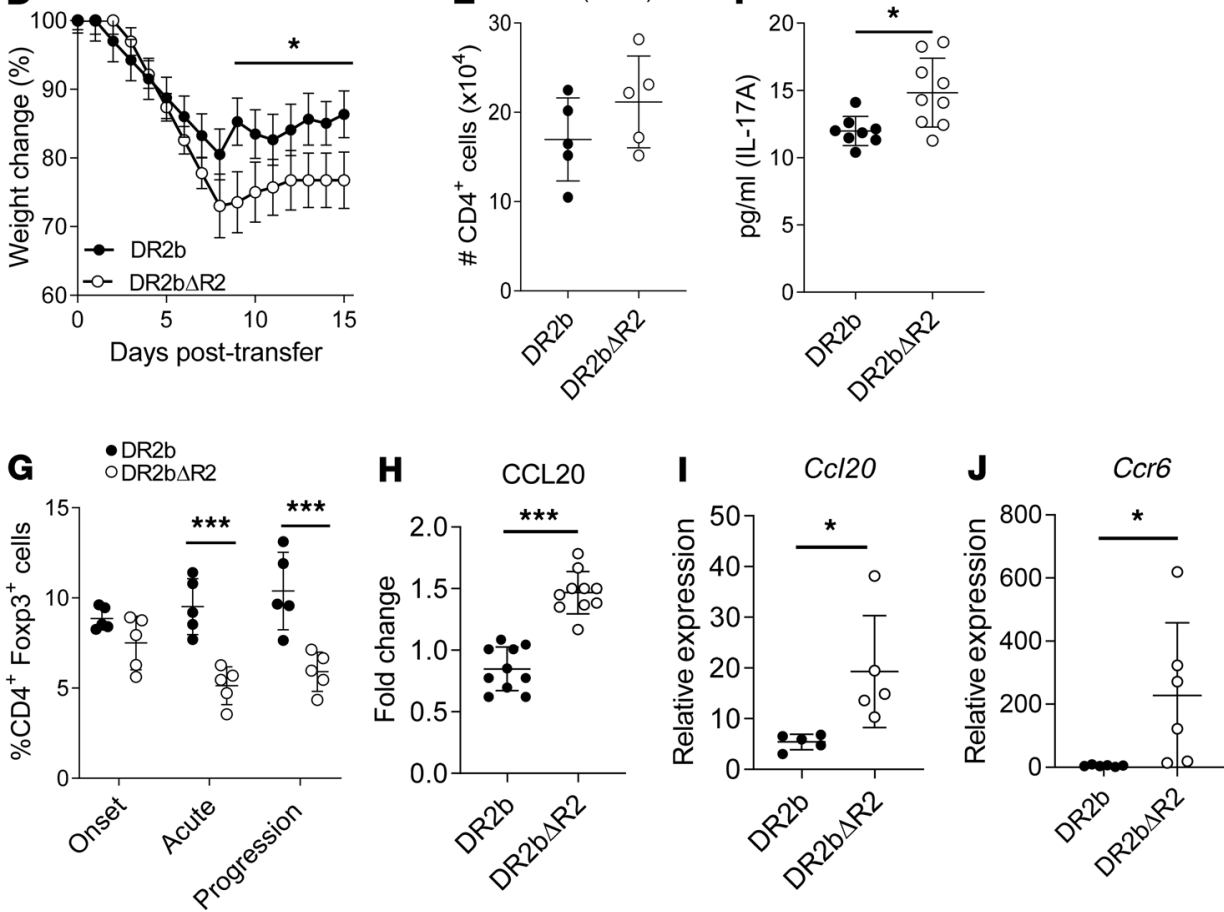

Figure 4. Development of progressive EAE and increased expression of CCL2O in DR2bAR2 recipient mice. (A) Schematic showing passive EAE induction of DR2b and DR2b $\triangle R 2$ mice (recipients) by transferring $\mathrm{MOG}_{35-55}$-reactive T cells obtained from DR2b donors and expanded in vitro. (B) Clinical signs of EAE, (C) clinical signs of ataxia, and (D) change in weight were monitored daily in $\mathrm{DR} 2 \mathrm{~b}$ and $\mathrm{DR} 2 \mathrm{~b} \triangle \mathrm{R} 2$ recipient mice after induction of adoptive transfer EAE with DR2b WT T cells. Representative data from 6 (B and C) and 3 (D) independent experiments with $n=4-8$ mice per group. (E) CD4 ${ }^{+} \mathrm{T}$ cell numbers in CNS at acute disease stage. Representative data from 3 independent experiments with $n=3-5$ mice per group. (F) Concentration of IL-17 in whole CNS tissue homogenate at acute disease. Pooled data from 3 independent experiments, $n=8$ for DR2b and $n=9$ for DR2b $\Delta R 2$ recipient mice. (G) Frequencies of CD4 ${ }^{+}$Foxp3 ${ }^{+}$ T cells in CNS at onset (day 7 after transfer), acute (day 12 after transfer), and disease progression phase (day 15 after transfer). Representative data from 3 independent experiments with $n=3-5$ mice per group. (H) Fold change of CCL20 in whole CNS tissue homogenate at days 12-15 after transfer (acute to progression phase) relative to the experimental mean expression. Pooled data of 3 independent experiments. $n=10$ mice per group. (I) Ccl20 mRNA expression in CNS tissue homogenate at acute disease measured by real-time PCR (qPCR). Representative data from 3 independent experiments with $n=3-5$ per group. (J) C Cr6 mRNA expression in CNS tissue homogenate at the progression phase measured by qPCR. Pooled data from 2 independent experiments with $n=6$ per group. Statistical significance was determined by 2-way ANOVA and for multiple comparisons with Holm-Šídák correction (B-D) or Student's 2-tailed $t$ test with Welch's correction $(\mathrm{E}-\mathrm{J}) .{ }^{*} P \leq 0.05$; and ${ }^{* *} P \leq 0.001$. Error bars indicate mean \pm SD.

with IFN- $\gamma$ and IL-17 reaching statistical significance at disease onset (Figure 2, D-F). However, the frequencies of cytokine-producing $\mathrm{T}$ cells were comparable at acute phase (days 12-15 after immunization) and during EAE disease progression (days 16-25 after immunization) (Figure 2, D-F), despite the progressive increase in disease severity over the disease course in DR2b $\Delta \mathrm{R} 2$ mice (Figure 1, D and E). Nevertheless, proliferation of $\mathrm{CD} 4^{+} \mathrm{T}$ cells isolated from $\mathrm{DR} 2 \mathrm{~b} \Delta \mathrm{R} 2$ mice with $\mathrm{EAE}$ was decreased during EAE progression compared with controls (Figure $2 \mathrm{G}$ ), corresponding to the modestly reduced $\mathrm{T}$ cell percentages and reduced proliferation noted in naive animals (Figure 2B). Similar to naive animals, Annexin 
A

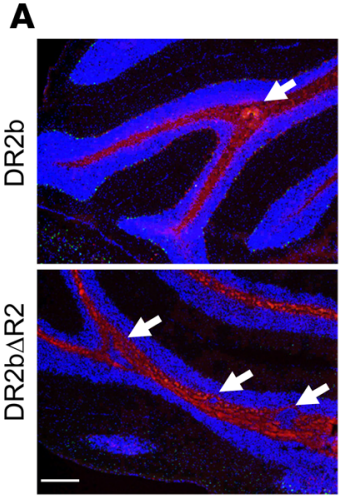

C
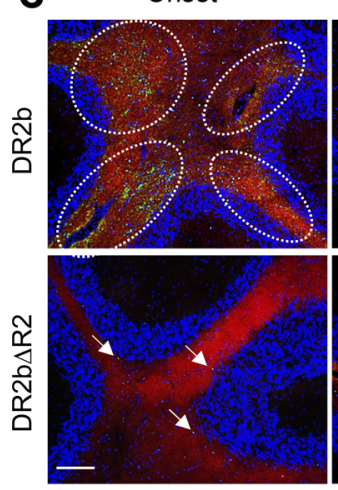

D

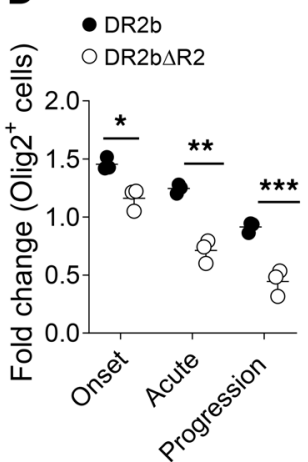

Myelin

DAPI

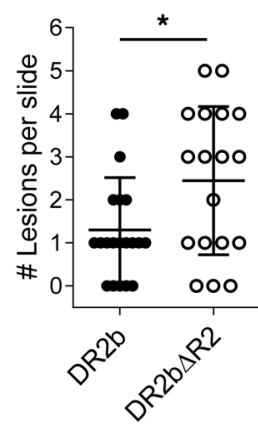

Acute
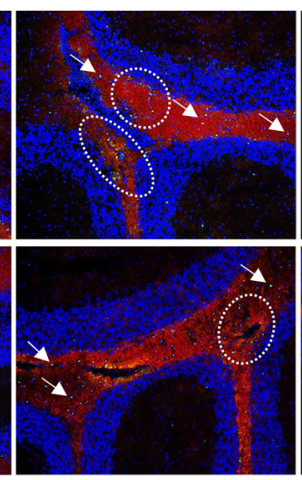

E

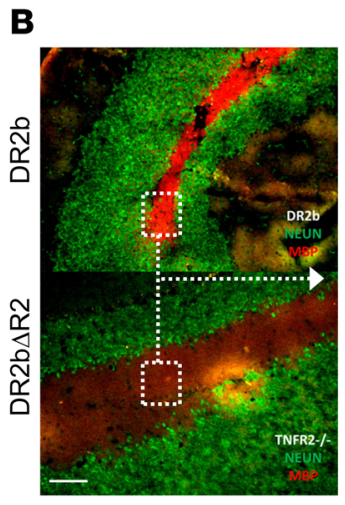

Myelin

NeuN

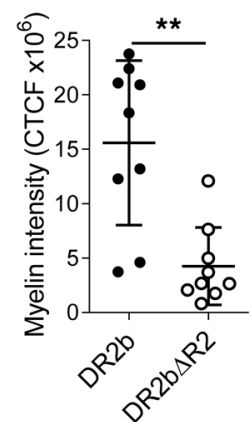

Progression

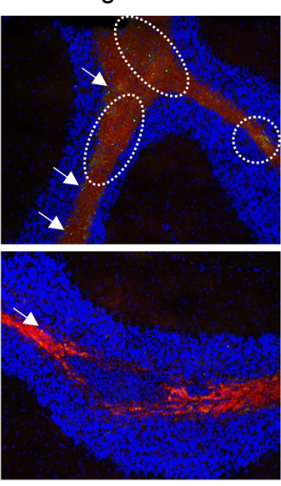

Myelin

Olig2

DAPI

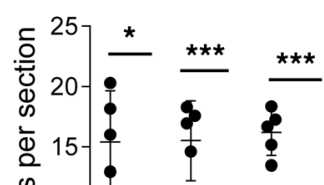

- DR2b

- DR2b $\Delta R 2$
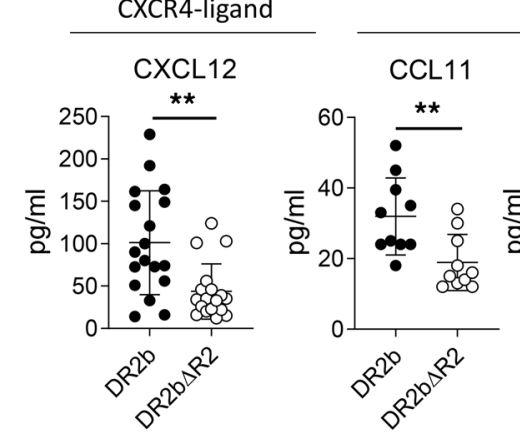

CCR3-ligands

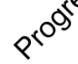


A

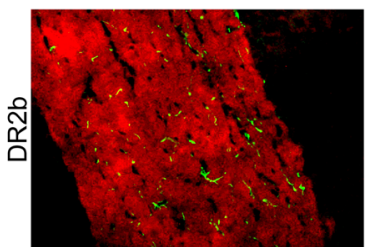

B
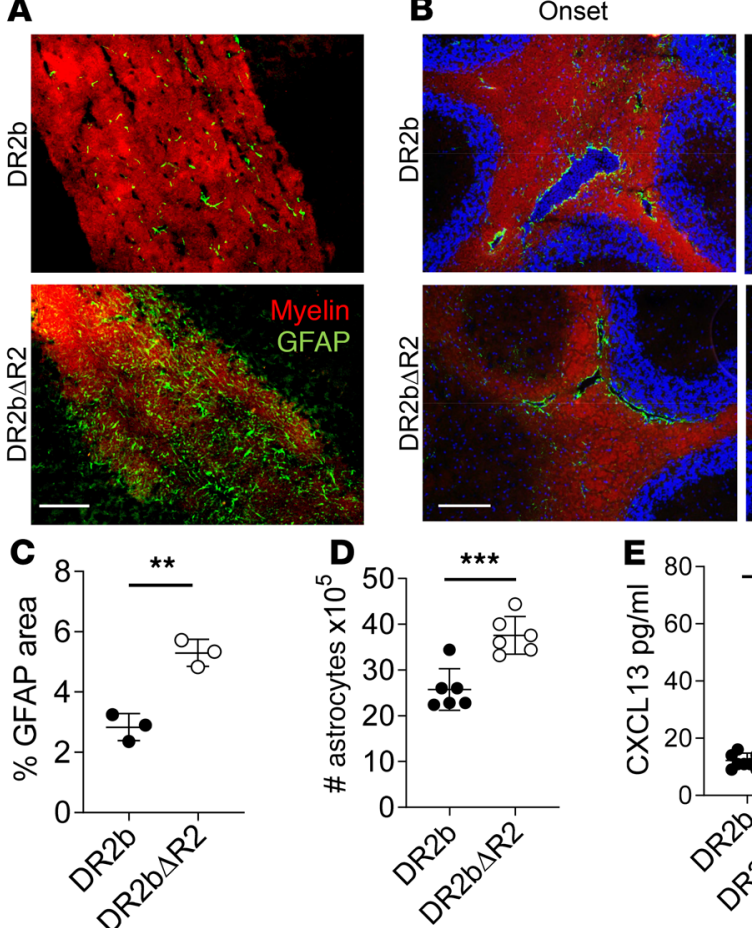

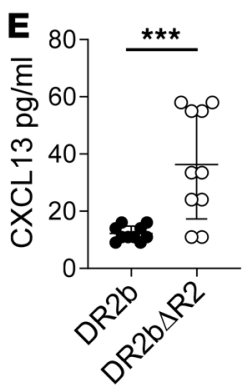

Acute
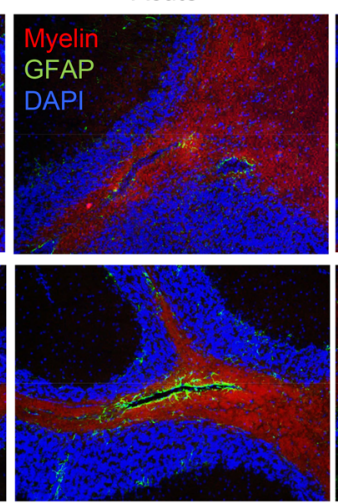

Progression
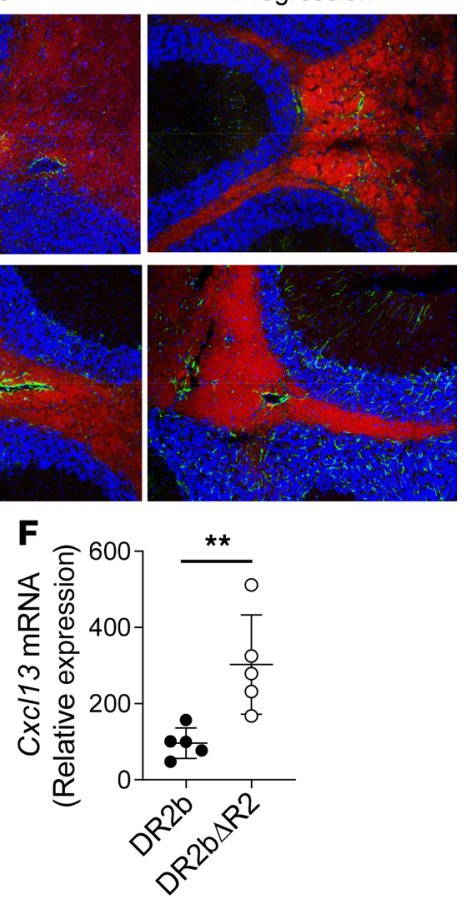

\section{G $\bullet \mathrm{DR} 2 \mathrm{~b}$}
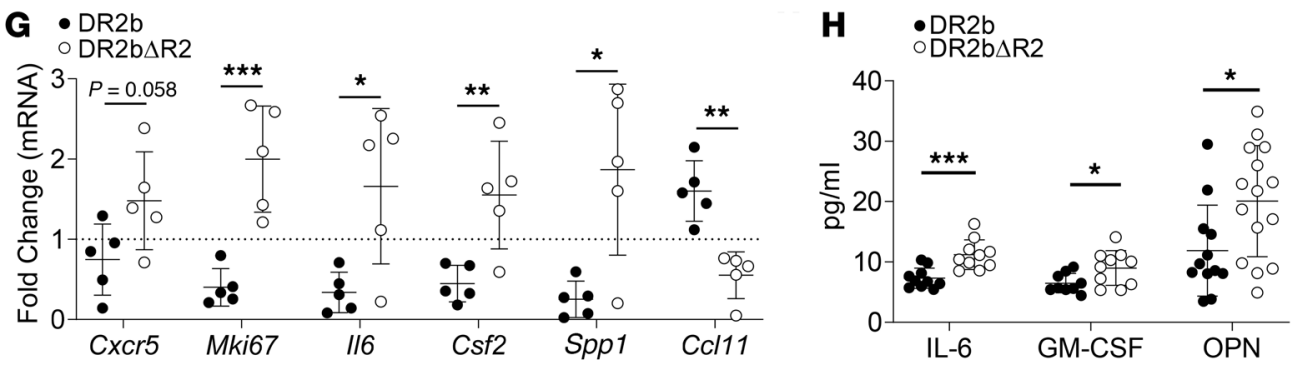

Figure 6. CNS TNFR2 limits chronic astrogliosis and astrocyte activation. Passive EAE induced via adoptive transfer of $\mathrm{MOC}_{35-55}$-reactive T cells from DR2b donors into DR2b and DR2b $\Delta R 2$ recipients. (A) Representative IF staining of cerebellum sections analyzed at the progression phase of disease, showing myelin stain (FluoroMyelin, red) and astrocytes (GFAP, green). Scale bar: $50 \mu \mathrm{m}$. (B) Representative IF staining of cerebellum sections analyzed at onset (top), acute phase (middle), and progression phase (bottom) of disease, showing myelin stain (FluoroMyelin, red), astrocytes (GFAP, green), and nuclear stain (DAPI, blue). Scale bar: $200 \mu \mathrm{m}$. Representative data from 4 independent experiments with 3-6 mice per group (A and B). (C) Percentage of GFAP-stained area per cerebellum as in $\mathbf{B}$, analyzed at the progression phase of disease. Representative data of 3 independent mice per group. (D) Number of astrocytes during EAE progression phase. Data from 2 independent experiments, $n=8$ per group. (E) Concentration of CXCL13 in whole CNS tissue homogenate at acute disease. Pooled data of 3 independent experiments, $n=10$ mice per group. (F) mRNA expression level of Cxc/13 in whole CNS tissue homogenate at acute disease. Representative data of 3 independent experiments, $n=3-5$ mice per group. (C) Astrocytes were isolated at acute phase and disease progression phase and analyzed for mRNA expression as indicated. Shown are fold changes relative to the experimental mean expression per each gene. Representative data of 2 experiments (Cxcr5 and Mki67) or 3 experiments (II6, Csf2, Ssp1, Cc/11) with $n=5-8$ mice per group. (H) Concentration of IL-6, GM-CSF, and osteopontin (OPN) in whole CNS tissue homogenate at acute disease. Data pooled from 3 independent experiments, $n=6-10$ mice per group. Statistical significance was determined by Student's 2-tailed $t$ test with Welch's correction and multiple comparisons with Holm-Šídák correction (E). ${ }^{*} P \leq 0.05,{ }^{* *} P \leq$ 0.01 , and ${ }^{* *} P \leq 0.001$. Error bars indicate mean \pm SD.

$\mathrm{V}^{+} \mathrm{CD}^{+} \mathrm{T}$ cells were not increased during EAE (Supplemental Figure 2A), indicating that the modest decrease in $\mathrm{T}$ cell numbers was not due to apoptosis. Additionally, we did not observe deviation of $\mathrm{T}$ cell responses toward a Th2 cell phenotype (Supplemental Figure 2B).

Thus, the results showed that TNFR2 deficiency had a modest effect on proliferation, clonal expansion, and cytokine profiles of HLA-DR2b-restricted $\mathrm{MOG}_{35-55}$-specific CD4 ${ }^{+} \mathrm{T}$ cells compared with WT $\mathrm{DR} 2 \mathrm{~b}$ T cells. The results suggested that TNFR2 signaling on conventional $\mathrm{CD} 4^{+} \mathrm{T}$ cells was dispensable for the disease phenotype observed in DR2b $\Delta \mathrm{R} 2$ mice. 
Foxp $3^{+}$Treg cells are critical to control pathogenic $\mathrm{CD}^{+} \mathrm{T}$ cell inflammation. TNFR2 was reported to promote Treg cell development and to augment Treg cell suppressive effector functions during EAE (32). In line with these reports, we found that Foxp $3^{+}$Treg cells were significantly reduced during EAE progression in DR2b $\Delta \mathrm{R} 2$ animals (Figure $2 \mathrm{H}$ ). However, the percentages of Foxp3 ${ }^{+}$Treg cells in naive DR2b and DR $2 b \Delta R 2$ mice were comparable (Figure 2I). These data suggest that TNFR2 may be important for Foxp $^{+}$Treg cell functions also in the context of human HLA-DR2b molecules during EAE. In support of this view, the serum levels of IL-10 were significantly decreased in DR $2 b \Delta R 2$ animals compared with $\mathrm{DR} 2 \mathrm{~b}$ controls during the progression phase of EAE, while serum levels of IL-17, GM-CSF, and TNF were significantly increased (Figure $2 \mathrm{~J}$ ).

TNFR 2 attenuates Th17 cell responses in the CNS of DR $2 b$ mice and ameliorates pathology. Next, we asked whether T cell responses in the CNS were affected by the absence of TNFR2 and whether this contributed to the development of progressive EAE. As shown in Figure 3A the frequencies of CNS-infiltrating Th17 cells were significantly higher in $\mathrm{DR} 2 \mathrm{~b} \Delta \mathrm{R} 2$ animals at the acute and progression phases of EAE compared with DR2b mice. However, frequencies of IFN- $\gamma$ - and GM-CSF-producing T cells were comparable between the groups (Figure 3, B and C). Notably, Foxp3 ${ }^{+}$Treg cell frequencies were significantly decreased after disease onset in the CNS of DR2b $\Delta$ R2 mice (Figure 3D), which may explain in part the increase in CNS Th17 reactivity (33).

In the CNS, Th17 cells amplify the inflammatory cascade by a variety of cellular mechanisms to promote tissue destruction and demyelination (34). Accordingly, the number of CNS lesions was significantly increased in $\mathrm{DR} 2 \mathrm{~b} \triangle \mathrm{R} 2 \mathrm{EAE}$ mice (Figure 3E and Supplemental Figure 3, A-C). An increased number of lesions and immune cell infiltrates was found during EAE in $\mathrm{DR} 2 \mathrm{~b} \triangle \mathrm{R} 2$ mice across different $\mathrm{CNS}$ regions, including spinal cord, cerebellum, brainstem, and periventricular regions (Supplemental Figure 3, A-C), which was accompanied by a significant reduction of myelin staining in cerebellar white matter of these animals during EAE (Figure $3 \mathrm{~F}$ and Supplemental Figure 3D). The decrease in myelination was specific for the EAE condition because no significant differences were noted in naive animals (Figure 3G). Together, the data showed that TNFR2 signaling dampened Th17-mediated pathology in the CNS and ameliorated demyelination during EAE.

T cell-extrinsic TNFR2 signaling curtails progressive EAE, increases CNS Tregs, and modulates CCL20 expression. TNFR2 is expressed not only by T cells but also by other cells implicated in the pathogenesis of EAE, for example endothelial cells, microglia, and astrocytes (35). Therefore, to discriminate between the role of TNFR2 expressed by T cells versus other cell populations on disease pathology, EAE was induced by adoptive transfer of myelin-reactive $\mathrm{T}$ cells from DR2b donor mice (TNFR2 $2^{+/+}$) to DR2b or DR2b $\Delta$ R2 recipient mice (Figure $4 \mathrm{~A})$.

Importantly, $\mathrm{DR} 2 \mathrm{~b} \Delta \mathrm{R} 2$ recipient mice, but not $\mathrm{DR} 2 \mathrm{~b}$ littermates, developed progressive EAE characterized by a steady increase of clinical EAE scores, cerebellar ataxia, and weight loss (Figure 4, B-D). The clinical features recapitulated EAE induced via active immunization with myelin antigen (Figure 1, D-F). Thus, these findings support an important role for T cell-extrinsic TNFR2 expression in restraining EAE progression in DR2b mice.

To further address this question, we analyzed CNS CD4 ${ }^{+} \mathrm{T}$ cells and Tregs, as well as inflammatory cytokines and chemokines, after induction of adoptive EAE. Consistent with our earlier findings in active $\mathrm{EAE}$, the number of $\mathrm{CD} 4^{+} \mathrm{T}$ cells in the CNS was only marginally higher in DR2b $\Delta \mathrm{R} 2$ recipient mice, and the differences were not statistically significant (Figure 4E). Nevertheless, CNS levels of IL-17 were significantly higher in DR2b $\triangle \mathrm{R} 2$ recipient mice (Figure 4F). Furthermore, the number of Treg cells in the CNS of $\mathrm{DR} 2 \mathrm{~b} \Delta \mathrm{R} 2$ recipient mice was significantly decreased at acute phase and during the progressive phase of disease (Figure 4G). Thus, the data indicated increased Th17-mediated immunopathology in the absence of TNFR2 signaling also in the adoptive transfer EAE model, which was not properly restrained by Treg cells and independent of TNFR2 expression by the encephalitogenic T cells.

Recruitment of Th17 cells to the CNS is promoted via the expression of CCR6, the CCL20 chemokine receptor (36). Therefore, we asked whether the levels of CCL20 and CCR6 were increased in this model. Indeed, we detected a significant increase in the expression of CCL20 protein and Ccl20 and Ccr6 mRNA levels in the CNS of DR2b $\Delta R 2$ mice with adoptive transfer EAE (Figure 4, H-J). These data suggest that $\mathrm{T}$ cell-independent expression of TNFR2 has important implications for regulation of CCL20 in the inflamed CNS to promote CCR6 ${ }^{+}$Th17 cell recruitment.

Taken together, the results showed that $\mathrm{T}$ cell-extrinsic TNFR2 signaling curtailed progressive EAE induced by adoptive transfer of HLA-DR2b-restricted TNFR2 ${ }^{+/+}$Th17 cells, potentially by regulating CNS Treg cells and expression of CCL20. 
TNFR2 is important to maintain CNS myelination and OPCs in HLA-DR2b mice. Our results showed that expression of TNFR 2 by host cells rather than by adoptively transferred T cells had an important effect on ameliorating EAE disease severity and progression. To further elucidate the underlying mechanisms, we examined CNS histopathology of $\mathrm{DR} 2 \mathrm{~b} \Delta \mathrm{R} 2$ or $\mathrm{DR} 2 \mathrm{~b}$ mice following passive EAE induction. In accordance with the increased disease severity of $D R 2 b \Delta R 2$ recipient animals compared with DR2b mice (Figure 4, B-D), we found a significant increase in CNS lesions accompanied by a significant reduction of myelin staining intensity, including in the cerebellum (Figure 5, A and B).

Previous studies demonstrated that TNFR2 expression is critical for promoting remyelination in the corpus callosum via OPCs in the cuprizone model of demyelination and in $\operatorname{EAE}(15,16,19)$. Conceivably, TNFR2 could also play a central role in promoting myelin repair in patients with MS. Importantly, progressive MS is frequently associated with cerebellar pathology (30), similar to the clinical signs (cerebellar ataxia) and pathology found in DR2b $\triangle \mathrm{R} 2 \mathrm{EAE}$ mice. Therefore, we examined remyelination and OPC numbers in the cerebellum in this model. As shown in Figure 5C, the number of Olig2 ${ }^{+}$OPCs in the cerebellum was significantly reduced in $\mathrm{DR} 2 \mathrm{~b} \Delta \mathrm{R} 2$ recipient mice during EAE. Flow cytometry analysis corroborated the reduction in Olig2 ${ }^{+} \mathrm{OPCs}$ in the CNS of DR2b $\Delta \mathrm{R} 2$ recipient mice over the disease course (Figure 5D). Notably, the most profound differences in Olig2 ${ }^{+}$OPCs were observed during the progression stage of EAE (Figure 5, C and D). In contrast, we did not observe differences in Olig2 ${ }^{+}$OPC numbers between naive DR2b and DR2b $\Delta R 2$ mice (data not shown). Thus, our results reveal that TNFR2 regulates cerebellar OPCs.

TNFR2-dependent expression of CXCL12 is critical for driving proliferation and differentiation of OPC via CXCR4 (16). Consistent with this notion, we observed a significant decrease in CXCL12 expression in DR2b $\triangle \mathrm{R} 2 \mathrm{CNS}$ tissue at acute and progressive phases of disease (Figure 5E). However, we did not observe significant differences in the expression of Cxcr4 (Supplemental Figure 4A). In addition to CXCR4, OPCs also express CCR3, which can regulate their function (37). However, similar to Cxcr4, no significant differences were observed in the expression of $C c r 3$ (Supplemental Figure 4B). Conceivably, changes in the mRNA expression levels of $C x c r 4$ or $C c r 3$ may have been below the level of detection. Therefore, we determined the expression of CCR3 ligands in CNS tissue during EAE, including CCL5, CCL7, and CCL11 $(37,38)$. The results showed that in DR $2 b \triangle \mathrm{R} 2 \mathrm{CNS}$, the protein expression levels of CCL11 and CCL7 were significantly reduced, whereas CCL5 expression was similar (Figure 5E).

Together, these data suggest that TNFR2 signaling is essential to promote myelin repair by regulating OPC numbers, potentially via CXCL12/CXCR4 and/or CCL11/CCR3 in progressive EAE.

TNFR 2 restrains cerebellar astrogliosis in EAE. Astrocytes are involved in key aspects of neuroinflammation and have been reported as central players in modulating chronic CNS inflammation and MS progression (39-41). TNF is a potent inducer of activation of astrocytes, which express both TNFR1 and TNFR2 (42). Additionally, astrocytes play key roles in remyelination by regulating OPC and oligodendrocyte functions, including secretion of CXCL12 in a TNFR2-dependent manner (16). Moreover, astrocytes are the primary source of CCL20 and CCL11 $(43,44)$, which were also significantly altered in the CNS of DR2b $\Delta$ R2 mice (Figure 4, $\mathrm{H}$ and I; and Figure 5E, respectively). Thus, we investigated whether TNFR2 signaling by astrocytes played a role in modulating disease progression in DR $2 b \Delta R 2$ mice. For this, we induced EAE by adoptive transfer of $M_{35}-55$-reactive $T$ cells from $D R 2 b$ mice to DR2b and DR2b $\Delta R 2$ mice and determined the presence of activated $\mathrm{GFAP}^{+}$astrocytes in the CNS. Strikingly, astrocytes accumulated in large numbers surrounding white matter tracts during EAE disease progression in DR2b $\Delta \mathrm{R} 2$ recipients but not in DR2b control mice (Figure 6A). To further investigate the kinetics of astrocyte accumulation during EAE, we determined their presence at onset, acute phase, and during EAE disease progression. We observed that the levels of reactive astrocytes in lesions at disease onset were comparable between DR2b and DR2b $\Delta$ R2 mice (Figure $6 B$ ). However, examination of astrocytes showed that they notably decreased in white matter tracts during acute and progression phases of EAE in DR2b recipients, whereas their numbers remained strikingly high in $\mathrm{DR} 2 \mathrm{~b} \Delta \mathrm{R} 2$ recipients and they developed chronic astrogliosis (Figure 6, B and C). Correspondingly, the number of astrocytes was significantly increased in the CNS of DR2b $\Delta R 2$ recipients during the progression phase (Figure 6D).

Injured neurons can upregulate CXCL13 to activate CXCR5 ${ }^{+}$astrocytes (45). Along this line, we noted a significant increase in the expression of CXCL13 in CNS tissue of DR2b $\Delta R 2$ recipients (Figure 6, E and F). Analysis of astrocytes isolated from EAE recipient mice at acute phase revealed that DR2b $\Delta R 2$ astrocytes expressed elevated mRNA levels of Cxcr5 and Mki67 (Ki-67) (Figure 6G). These data suggested 
that $\mathrm{DR} 2 \mathrm{~b} \triangle \mathrm{R} 2$ astrocytes were activated in a CXCL13/CXCR5-dependent manner and proliferated more extensively than DR2b astrocytes, thereby resulting in chronic astrogliosis.

Activated astrocytes can promote tissue damage via expression of proinflammatory mediators. Importantly, astrocytes isolated from $\mathrm{DR} 2 \mathrm{~b} \Delta \mathrm{R} 2$ recipients showed significantly higher expression of $I l 6$ (IL-6), Csf2 (GM-CSF), and Spp1 (OPN) (Figure 6G). Similarly, protein expression of IL-6, GM-CSF, and OPN was significantly higher in the CNS of DR2b $\Delta$ R2 recipients (Figure $6 \mathrm{H}$ ), suggesting that inflammatory cytokine production by astrocytes is increased in the absence of TNFR2-mediated signaling. Interestingly, we observed a significant decrease in Ccl11 expression in astrocytes isolated from $\mathrm{DR} 2 \mathrm{~b} \Delta \mathrm{R} 2$ recipient mice (Figure $6 \mathrm{G}$ ), which corresponded to its significantly decreased protein expression in the CNS (Figure 5E). Astrocyte CCL11 can promote remyelination and increases blood-brain barrier (BBB) integrity (46); thus our data suggest that astrocyte-protective functions are upregulated by TNFR2 signaling.

Taken together, the data suggest that TNFR2 modulated the CNS inflammatory milieu at least in part by regulating astrocyte activation via the CXCR5/CXCL13 axis to promote astrocyte proliferation and proinflammatory functions. Moreover, we posit a novel mechanism whereby TNFR2-dependent expression of astrocyte CCL11 promotes remyelination via CCR3 ${ }^{+}$OPCs. Our data identify TNFR2 as a key factor for limiting pathogenic astroglia functions during neuroinflammation.

\section{Discussion}

MS has both genetic and environmental predispositions, including HLA-DR2b (28). Importantly, most patients with MS eventually develop progressive MS. However, the potential mechanisms driving progressive MS and the potential contribution of HLA-DR2b to MS progression are not well understood.

The TCR-MHC complex plays a key role in thymic T cell development and in guiding $\mathrm{T}$ cell subset differentiation and effector responses upon $\operatorname{Ag}$ encounter $(5,6)$. Moreover, HLA molecules might have disease-promoting roles by supporting the generation of pathogenic autoreactive $\mathrm{T}$ cells, or alternatively, they may contribute to induction of tolerance by depleting self-reactive clones or generating Treg cells. For instance, HLA-DR2b promotes development of autoantigen-specific pathogenic Th1 and Th17 cells in Goodpasture disease, whereas HLA-DR1 promotes induction of autoantigen-specific Treg cells (47). Thus, investigation of neuroinflammatory disease in mice expressing HLA-DR2b molecules may provide information not ordinarily obtainable from conventional mouse models of EAE. Accordingly, here we show that HLA-DR2b promoted increased frequencies of encephalitogenic Th17 cells and impaired Treg cell formation compared with B6 animals in EAE. Future studies should investigate T cell immunity against additional neuroantigens in this model and their role for progressive disease.

Gene admixture studies have suggested a key role for TNF and its receptors TNFR1 and TNFR2 in the pathogenesis of autoimmune diseases, including MS $(23,48)$. Although TNFR1 is critical for EAE development, TNFR2 appears primarily protective in the context of autoimmune diseases $(49,50)$. Conceivably, impaired TNFR2 signaling may modulate disease progression in MS as suggested by disease exacerbation of MS patients treated with TNF inhibitors (9) and development of CNS-demyelinating disease in antiTNF-treated patients (51).

In the present study, we used our $\mathrm{DR} 2 \mathrm{~b} \Delta \mathrm{R} 2$ mouse model to elucidate potential immune-protective mechanisms of TNFR2 in the context of EAE induced by HLA-DR2b-restricted encephalitogenic $\mathrm{T}$ cells. Remarkably, we found that $\mathrm{DR} 2 \mathrm{~b} \Delta \mathrm{R} 2$ mice developed progressive clinical features, including paralysis and cerebellar ataxia.

Astrocytes are the most abundant cell type in the mammalian CNS and are central regulators of neuronal homeostasis and activity, as well as of innate and adaptive immune responses. Depending on timing and context, astrocyte activity may exacerbate CNS inflammation and tissue damage or promote immunosuppression and tissue repair (52-54). Under pathological conditions, astrocytes can promote pathogenesis and neuroinflammation via several mechanisms, including modulating BBB integrity, activation of autoreactive $\mathrm{T}$ cells, secretion of chemokines to promote CNS leukocyte infiltration, and support of pathogenic crosstalk with microglia cells $(55,56)$. Reactive astrocytes can directly induce neuronal damage and demyelination through increased expression of proinflammatory mediators, such as nitric oxide, or decreased expression of protective molecules, such as glutamine synthetase, that restrict neurotoxicity (57). Importantly, TNF is mitogenic for astrocytes and strongly induces their activation and proliferation (58). Both TNFR1 and TNFR2 are expressed by astrocytes, though signaling through the respective receptors 
results in different outcomes (42). Herein we show that lack of TNFR2 signaling resulted in chronic CNS astrogliosis and that $\mathrm{DR} 2 \mathrm{~b} \Delta \mathrm{R} 2$ astrocytes proliferated more extensively and expressed increased levels of proinflammatory mediators, including IL-6, GM-CSF, and OPN. On the other hand, DR2b $\Delta$ R2 astrocytes expressed less CCL11, a chemokine that can regulate OPC and myelination in a CCR3-dependent manner and may also be important to maintain BBB integrity $(37,46)$. These data strongly suggest that TNF signaling via TNFR2 is critical for limiting the inflammatory capacity of astrocytes.

CXCL13 expression is significantly increased in active MS lesions and promotes B cell-mediated pathology (59). Furthermore, recent data showed that CNS CXCL13 can also activate CXCR5 ${ }^{+}$astrocytes to facilitate astrocyte-mediated pathogenesis of neuropathic pain (45). Accordingly, our data show that TNFR2 signaling limits astrocyte CXCR5 expression, as well as CNS CXCL13 expression. Interestingly, a loss-of-function polymorphism allele of CXCR5 (rs630923) was identified as a risk-lowering factor for MS (60). This polymorphism results in lower transcription of CXCR5 upon NF- $\mathrm{kB}$-inducing signals, such as TNF. Interestingly, CXCL13 is produced mainly in actively demyelinating MS lesions but not in inactive lesions (61). Therefore, it is conceivable that in progressive MS, CXCL13 activates astrocytes and endows them with proinflammatory functions (45). Notably, CXCL13 is highly expressed by injured neurons, though it can also be expressed by stromal cells and microglia $(45,59)$. Thus, our data suggest that expression of CXCL13 is suppressed by TNFR2 signaling and not TNFR1. Furthermore, our data show that TNFR2 signaling impedes CNS CXCL13 and astrocyte CXCR5 expression, therefore harnessing CNS pathogenicity and chronic astrogliosis. Thus, we posit a novel TNFR2-mediated regulatory mechanism in which CXCL13 activates astrocytes in a CXCR5-dependent manner to promote disease progression. Future studies will be required to further elucidate the role of the CXCL13/CXCR5 axis in astrocyte-mediated pathology and to identify the critical cellular sources of CXCL13 and its regulation by TNFR2.

Reactive astrocytes express increased levels of IL-6, GM-CSF, OPN, and TNF during the chronic stage of EAE, resulting in astrogliosis, neuronal damage, and axonal pathology $(39,62,63)$. Furthermore, astrocyte IL-6 facilitates CNS entry of T cells by increasing BBB permeability and increased adhesion molecule expression by endothelial cells (64), and it activates microglial cells to become proinflammatory and promote damage to oligodendrocytes and axons $(64,65)$. Our data indicate that TNFR2 regulates or suppresses the expression of IL-6, whereas TNFR1 may be important for its induction.

During the chronic phase of EAE, astrocytes express high levels of GM-CSF, which is dependent on autocrine signaling by lactosylceramide, which appears to be critical for astrogliosis and EAE progression (39). The GM-CSF receptor is upregulated on activated astrocytes and microglia cells in EAE and MS to promote damage to neurons and oligodendrocytes (66). Moreover, GM-CSF can sustain neuroinflammation via the induction of an IL-23 and Th17 cell positive feedback loop (67). Because both IL-6 and GM-CSF (via IL-23 induction) are critical for Th17 cell development, it is conceivable that reactive astrocytes contribute to sustaining in situ Th17 cell pathogenicity and chronic neuroinflammation. However, under conditions of chronic astrocyte activation, sustained production of inflammatory mediators such as IL-6 may suffice to promote disease progression even in the absence of significant numbers of T cells in lesions. Along these lines, inactive lesions that are typical for progressive MS are dominated by the presence of innate immune and glial cells but not $\mathrm{T}$ cells (68).

OPN is highly expressed in demyelinating lesions in MS and EAE, and some studies suggest that its levels correlate with disease severity $(69,70)$. OPN may promote EAE progression by inhibiting IL-10 and enhancing IFN- $\gamma$ expression by myelin-reactive T cells (69). Additionally, OPN can promote the requirement and function of Th17 cells $(71,72)$. Astrocytes upregulate the expression of OPN during EAE and thus can assist in sustaining Th17-mediated pathology.

Lastly, astrocytes can also promote CNS protection and remyelination during neuroinflammation, for instance by recruiting and supporting OPC maturation and myelination via TNFR2-dependent expression of CXCL12 $(16,73)$. Our results support this view but also suggest the CCR3/CCL11 axis as a potentially novel TNFR2-dependent neuroprotective mechanism that may regulate OPC function and remyelination during chronic inflammation.

A key remaining question is what the initial signals are for chronic astrocyte activation. Along this line, Th17 cell cytokines and particularly IL-17 can enhance inflammatory functions of astrocytes (74), while CNS Treg cells are pivotal for suppressing astrogliosis and astrocytic damage $(75,76)$. Consistent with these reports, DR2b $\triangle$ R2 EAE mice showed an increase of CNS and serum Th17 cell responses, while Treg cells and IL-10 were significantly reduced. Notably, we found that HLA-DR2b positively regulated Th17 
cell differentiation, while negatively affecting Treg cell generation independent of TNFR2. These results are in line with other reports showing that autoimmune disease-associated HLAs can promote differentiation of autoantigen-specific pathogenic T cells $(77,78)$. Nonetheless, impaired TNFR2 signaling also favors differentiation of Th17 cells over Treg cells $(13,79,80)$. Thus, the expression of DR2b and lack of TNFR2 signaling could synergize in the development of chronic astrogliosis by inhibiting Treg cells and promoting a robust Ag-specific Th17 cell pool and its accumulation in the CNS.

Taken together, our data support that TNFR2 signaling is critical for restraining pathogenic astrocyte effector functions. Thus, targeting proinflammatory pathways in astrocytes via TNFR1 while promoting their protective properties via TNFR2 could be a novel and effective approach for preventing progression of MS.

\section{Methods}

Mice. HLA-DR2b-Tg (DRB1*15:01-Tg) mice $(81,82)$ and TNFR2 ${ }^{-/-}$mice $(83)$ (The Jackson Laboratory

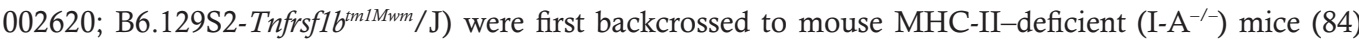
(The Jackson Laboratory 003584; B6.129S2- $H 2^{d \mathrm{dAbl}-\mathrm{Ea}} / \mathrm{J}$ ) to eliminate endogenous murine MHC-II genes. The offspring from each of the crosses were backcrossed to generate HLA-DR2 $\mathrm{b}^{+/+} \mathrm{I}_{-} \mathrm{A}^{-/-} \mathrm{TNFR}^{-/-}$mice, designated DR2b $\triangle R 2$. The expression of I-A, HLA-DR2b, and TNFR2 was verified by PCR and flow cytometry. C57BL/6 mice (The Jackson Laboratory 000664) and $\mathrm{TNF}^{-1-}$ mice (The Jackson Laboratory 003008) were purchased from The Jackson Laboratory. Mice were immunized after 6 weeks of age and supplemented with DietGel Recovery $\left(\mathrm{ClearH}_{2} \mathrm{O}\right)$ as needed during disease studies. All experiments included age- and sex-matched controls; littermate controls were used unless indicated otherwise in the text.

Genotyping and primers. DNeasy Blood \& Tissue Kit (Qiagen 69506) was used to extract genomic DNA from ear notches. DNA was amplified using GoTaq Green Master Mix (Promega M7133). The following primers were obtained from Invitrogen, Thermo Fisher Scientific: HLA-DR2b primers: forward, 5'-GTTTCCTGTGGCAGCCTAAGAGG-3'， and reverse, 5'-TCCACCGCGGCCCGCG-3'; mouse MHC-II, I-A primers: forward, 5'-GGGGTGGAATTTGACCTCTT-3', and reverse, 5'-TGGAGACATTGGCCAGTACA-3'; TNFR2-KO primers: forward, 5'-GCCCTGAATGAACTGCAGGACG-3', and reverse, 5'-CACGGGTAGCCAACGCTATGTC-3'; TNFR2-WT primers: forward, 5'-AGCTCCAGGCACAAGGGCG-3', and reverse, 5'-CCTCTCATGCTGTCCCGGA-3'; and HLA-DR2 primers: forward, 5'-AGCTCCAGGCACAAGGGCGGG-3' and reverse, 5'-CCTCTCATGCTGTCCCGGAAT-3'.

EAE induction. Active EAE was induced by subcutaneous (s.c.) injection of $100 \mu \mathrm{g} \mathrm{MOG}_{35-55}$ peptide (United Biochemical Research) in $50 \mu \mathrm{L}$ of CFA containing $5 \mathrm{mg} / \mathrm{mL}$ Mycobacterium tuberculosis $\mathrm{H} 37 \mathrm{Ra}$ (Difco Laboratories). Mice also received intraperitoneal injections of $200 \mathrm{ng}$ Bordetella pertussis toxin (List Biological) on days 0 and 2. For induction of passive EAE by adoptive transfer, donor mice were immu-

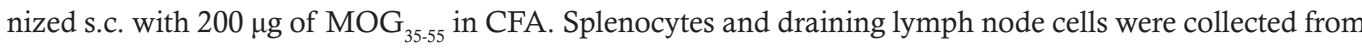
donor mice on day 9 and restimulated for 3 days at $37^{\circ} \mathrm{C}$ with $10 \mu \mathrm{g} / \mathrm{mL}$ of $\mathrm{MOG}_{35-55}$ peptide in complete DMEM containing $10 \%$ fetal bovine serum, $20 \mathrm{ng} / \mathrm{mL}$ of recombinant mouse IL-23 (R\&D Systems 1887 ML-010), and $10 \mu \mathrm{g} / \mathrm{mL}$ of anti-IFN- $\gamma$ monoclonal antibody (mAb) (Bio X Cell R4-6A2) to expand Th17 cells. Ten million to 12 million activated donor $\mathrm{T}$ cells were injected into the recipient mice.

EAE evaluation. Mice were assigned clinical scores for EAE according to the following scale: 0, no abnormality; 1, limp tail; 2, moderate hind limb weakness; 3 , complete hind limb paralysis; 4, quadriplegia or premoribund state; and 5 , moribund, death.

Ataxia evaluation. Mice were monitored and graded daily for clinical signs of ataxia using an adapted ataxia scoring system (85). Briefly, mice were evaluated daily in 3 ataxia categories: hind limb clasping, gait, and kyphosis on a scale of 0 (no clinical ataxia signs) to 3 (most severe clinical score). The final ataxia score represents the cumulative scores from all 3 categories and is on a scale of 0-9.

CNS tissue processing and immunofluorescence/immunohistochemistry staining. Mice were anesthetized with and perfused intracardially with cold HBSS and $4 \%$ paraformaldehyde. Brain and spinal cord tissues were then cryoprotected in O.C.T. compound (Thermo Fisher Scientific) and stored at $-80^{\circ} \mathrm{C}$. Brain and spinal cord tissue were procured and cut medial or horizontal to 8 - to $11-\mu \mathrm{M}$ sections using a Thermo Shandon cryostat. Slides were stained for myelin using FluoroMyelin Red (Invitrogen, Thermo Fisher Scientific, F34652) fluorescent myelin stain according to the manufacturer's guidelines and imaged using an Olympus BX41 microscope. Images were obtained using Olympus CellSens program (Olympus Life Science) and processed using Adobe Photoshop. The brightness and contrast were adjusted and were changed equivalently across all groups. Myelin fluorescence intensity was analyzed using ImageJ (NIH). During ImageJ 
analysis, images were adjusted to 8-bit, and exposure was adjusted to 0-20 across all groups. Three positive and 3 negative (background) measurements were taken per section. The fluorescence intensity per mouse was calculated as the average of 9 independent sections. Integrated density was subtracted from the area of the white matter of the cerebellum multiplied by the average of the background mean gray value to calculate the CTCF values, as described previously (86). Lesions were defined as visualized demyelinating areas along the myelin tracks containing cellular infiltrates (nuclear stains; DAPI \pm CD11b, CD4, CD45 stains). To determine percentage of glial fibrillary acidic protein (GFAP) area, the average fluorescence was calculated as the mean of 3 sections per brain as described previously (87).

Flow cytometry. Single-cell suspensions were obtained from spleen, lymph nodes, and brain tissues by mechanical disruption. Extracellular myelin was removed from all brain and spinal cord suspensions using myelin removal beads (Miltenyi Biotec), according to the manufacturer's instructions. Cell viability (exclusion of dead cells) was determined using Ghost Dye staining (Tonbo Biosciences) according to the manufacturer's protocol. For surface staining, cell suspensions were blocked with 1\% anti-mouse CD16/CD32 (eBioscience; 93) for 20 minutes on ice and then stained with fluorescently labeled anti-mouse antibodies for 45 minutes in PBS 1\% FBS (FACS buffer) at $4^{\circ} \mathrm{C}$. Following staining, cells were washed with FACS buffer, lysed for red blood cells (Beckman Coulter), and fixed using a fixative agent (eBioscience; 00-5523$00)$. Intracellular staining was performed following fixation and permeabilization. Samples were analyzed on a BD FACSAria II or LSRFortessa (BD Biosciences). Data were analyzed using BD FACSDiva (BD Biosciences) and FlowJo V10 data analysis software (FlowJo LLC).

Antibodies and staining reagents. The following antibodies were used to stain: CD4 (BD Biosciences; RM4-5), CD11c (eBioscience; N418), CD11b (eBioscience; M1/70), GR1 (BD Biosciences; RB6-8C5), TNFR2 (TNFRSF1B; R\&D Systems; FAB426A), HLA-DR2b (eBioscience; LN3), mouse MHC-II, I-A (BD Biosciences; AF6-120.1), Ly6G (BioLegend; 1A8), CD45 (eBioscience; 30-F11), Ki-67 (BD Pharmingen; B56), Foxp3 (eBioscience; FJK-16s), Olig-1 (Santa Cruz Biotechnology; K-14), Olig-2 (MilliporeSigma; 211F1.1 and MABN50A4), GFAP (MilliporeSigma; GA5), and NeuN (MilliporeSigma; A60). All antibodies were used according to the manufacturers' suggestions. For the detection of cell apoptosis, Annexin V Apoptosis Detection Kit (BD Pharmingen), containing FITC-conjugated anti-Annexin V and propidium iodide staining solution (nuclear stain), was used according to the manufacturer's guidelines. DAPI (MilliporeSigma) was used for nuclear staining in immunofluorescence/immunohistochemistry tissue staining.

Astrocyte isolation and quantification. Single-cell suspensions were obtained from whole CNS tissue using Neural Tissue Dissociation Kit (T) (Miltenyi Biotec 130-093-231). Astrocytes were isolated using antiACSA-2 MicroBead kit following the manufacturer's guidelines (Miltenyi Biotec 130-097-678). Following magnetic bead separation, cells were stained with anti-ACSA-2 antibody (clone IH3-18A3; Miltenyi Biotec) and analyzed by flow cytometry. Astrocyte purity was greater than $90 \%$. Astrocyte numbers per CNS were calculated as number of counted cells times percentage of astrocytes.

Real-time PCR and RNA extraction. Total RNA was extracted using RNeasy Lipid Tissue Mini Kit (Qiagen). The RNA was reverse-transcribed to cDNA using the cDNA reverse transcription kit (Life Technologies). Realtime PCR was performed with the CFX96 Touch Deep Well Real-Time PCR Detection System (Bio-Rad) using $\mathrm{RT}^{2}$ SYBR Green master mix (Qiagen) per the manufacturer's instructions. RT ${ }^{2}$ profiler PCR array for mouse chemokines and receptors and $\mathrm{RT}^{2} \mathrm{qPCR}$ primers for mouse genes were purchased from Qiagen, including for Cxcr5: NM_007551, Mki67: NM_001081117, I16: NM_031168, Csf2: NM_009969, Spp1: NM_001204201, Cc111: NM_011330, Cxc113: NM_018866, and Cxcr5: NM_007551. RNA and cDNA concentrations were estimated using a NanoDrop spectrophotometer. Transcript expression was normalized to GAPDH (NM_008084) and is represented as $2^{-\Delta \mathrm{CT}} \times 10,000$.

Serum collection. Samples were obtained from a single cheek puncture for each individual mouse. Whole blood samples were collected using BD Vacutainer SST Blood Collection Tubes (BD Worldwide 367381) for serum separation (88). Specimens were centrifuged at $1100 \mathrm{~g}$ for 20 minutes after clotting for 30 minutes.

Brain tissue lysate. Protein was extracted from whole mouse brain using the RIPA Lysis Buffer Kit (Santa Cruz Biotechnology SC-24948A) as per the manufacturer's protocol. Briefly, RIPA buffer was added to the cell pellet, incubated on ice for 5 minutes, and then centrifuged at $14,000 \mathrm{~g}$ for 15 minutes at $4^{\circ} \mathrm{C}$. The supernatant was collected and stored at $-80^{\circ} \mathrm{C}$ until further use.

Cytokine ELISPOT assay. Cytokine ELISPOT assay was performed and spots analyzed as described previously (82). In brief, ELISPOT plates (MilliporeSigma) were precoated with anti-mouse IFN- $\gamma \mathrm{mAb}$ (eBioscience, AN-18) and anti-mouse IL-17 mAb (Bio X Cell, 17F3). Splenocytes $\left(5 \times 10^{5}\right.$ cells per well) 
and brain-infiltrating mononuclear cells $\left(2 \times 10^{4}\right.$ to $5 \times 10^{4}$ cells per well) were restimulated with $\mathrm{MOG}_{35-55}$ peptide in HL-1 medium (Lonza) at $37^{\circ} \mathrm{C}$ for 24 hours. Biotinylated anti-mouse IFN- $\gamma$ mAb (eBioscience, R4-6A2) and anti-mouse IL-17 mAb (BioLegend, TC11-8H4) were then added overnight at $4^{\circ} \mathrm{C}$, followed by incubation with streptavidin-alkaline phosphatase (Invitrogen, Thermo Fisher Scientific) for 2 hours at room temperature and developing with BCIP/NBT Phosphatase Substrate (KPL). After plate developing, image analysis of spots was performed on a Series 2 Immunospot analyzer (Cellular Technology Limited). Results for antigen-specific spot-forming cells were normalized with a negative control containing peptide-free medium. All measurements were performed in duplicate or triplicate.

Enzyme-linked immunosorbent assay. Commercial mouse ELISA kits were purchased from Invitrogen, Thermo Fisher Scientific, including for IL-17 (catalog 88-7371-88), GM-CSF (catalog 88-7334-88), CXCL13 (catalog EMCXCL13), and IL-6 (catalog 88-7064-86), and from Ray-Biotech, including for OPN (catalog ELM-OPN) and IFN- $\gamma$ (catalog ELM-IFNg-1). All ELISA kits were used per the manufacturers' suggested protocols. For brain tissue specimens, 200-1000 $\mu \mathrm{g}$ of protein per sample was added to the corresponding well in each plate. For serum specimens, $40 \mu \mathrm{L}$ of serum and $60 \mu \mathrm{L}$ of sample diluent per sample were added to the corresponding well in each plate. Plates were read at OD450 $\mathrm{nm}$ for absorbance on a Synergy HT microplate reader (BioTek) and analyzed using BioTek Gen5 software.

Cytokine and chemokine multiplex assay (Bio-Plex). Brain tissue specimens were collected and analyzed for mouse cytokine and chemokine levels by a multiplex assay using the Bio-Rad Bio-Plex Pro Mouse Cytokine 23-plex Assay (m60009rdpd) and Bio-Plex Pro Mouse Chemokine Panel 33-Plex (12002231) per the manufacturer's protocol.

Statistics. The graphical abstract was created with BioRender (https://biorender.com). Statistical analysis, including Student's $t$ test and 1- or 2-way ANOVA, were performed using GraphPad Prism 8 and SigmaPlot 12 software. Statistical significance was determined as indicated in the text and figure legends. Unless otherwise indicated, an unpaired 2-tailed Student's $t$ test was performed. Gaussian distribution was assumed unless indicated otherwise. Where appropriate, statistical significance was corrected for multiple comparisons using Holm-Šídák method or for unequal SD using Welch's correction. A $P$ value less than 0.05 was considered significant. $P$ values are as follows: NS, not significant; ${ }^{*} P \leq 0.05 ;{ }^{* *} P \leq 0.01$; and ${ }^{* *} P \leq 0.001$.

Study approval. All animals were maintained in specific pathogen-free conditions in the American Association for Laboratory Animal Science-accredited facility at the University of Texas at San Antonio. All experiments were approved by the Institutional Animal Care and Use Committee of the University of Texas at San Antonio (UTSA) and performed in accordance with the relevant guidelines and regulations. Mice were fed and watered ad libitum.

\section{Author contributions}

IR, FGR, RAR, RRR, and SN performed the experiments and collected and analyzed the data. RAR and IR crossed and generated the DR2b $\Delta R 2$ colony. RAR, FGR, and SN maintained DR2b and DR2b $\Delta R 2$ mouse colonies. IR and TGF designed the experiments and reviewed the data. IR and TGF wrote and revised the manuscript. All authors reviewed and approved the manuscript. IR and TGF conceived the study. TGF supervised and financed the study.

\section{Acknowledgments}

This study was supported by grants G12MD007591 and NS084201 from the NIH (TGF) and grants RG5501 and RG1602 from the National Multiple Sclerosis Society (TGF).

The authors thank Bernard Arulanandam (UTSA), Neal Guentzel (UTSA), Astrid Cardona (UTSA), Chiung-Yu Hung (UTSA), Edu B. Suarez-Martinez (University of Puerto Rico at Ponce), and Niannian Ji (University of Texas Health Science Center San Antonio) for helpful critical suggestions and support. We thank Rishein Gupta (UTSA) for assistance with RT-PCR analysis and Rebecca Sosa (UCLA) for assistance with microscopy. We thank Ildiko Forsthuber (UTSA), the Research Centers in Minority Institutions program, UTSA Laboratory Animal Resources Center, and the Immunology Defense Core facilities at UTSA for assistance. FGR was partially supported by NIH National Institute of General Medical Sciences grant R25GM096955 (to Edu B. Suarez-Martinez). The authors would like to give special thanks to Babette Fuss (Virginia Commonwealth University) for critically reviewing this manuscript. 
Address correspondence to: Thomas G. Forsthuber, Department of Biology, University of Texas at San Antonio, Biotechnology, Sciences and Engineering Building room 3.250, 1 UTSA Circle, San Antonio, Texas 78249, USA. Phone: 210.458.5760; Email: thomas.forsthuber@utsa.edu.

1. Compston A, Coles A. Multiple sclerosis. Lancet. 2008;372(9648):1502-1517.

2. Goverman J. Autoimmune T cell responses in the central nervous system. Nat Rev Immunol. 2009;9(6):393-407.

3. Baldassari LE, Fox RJ. Therapeutic advances and challenges in the treatment of progressive multiple sclerosis. Drugs. 2018;78(15):1549-1566.

4. Barcellos LF, et al. Heterogeneity at the HLA-DRB1 locus and risk for multiple sclerosis. Hum Mol Genet. 2006;15(18):2813-2824

5. Gianfrani C, Pisapia L, Picascia S, Strazzullo M, Del Pozzo G. Expression level of $r$ isk genes of MHC class II is a susceptibility factor for autoimmunity: New insights. J Autoimmun. 2018;89:1-10.

6. Moran AE, Hogquist KA. T-cell receptor affinity in thymic development. Immunology. 2012;135(4):261-267.

7. Sedger LM, McDermott MF. TNF and TNF-receptors: from mediators of cell death and inflammation to therapeutic giants past, present and future. Cytokine Growth Factor Rev. 2014;25(4):453-472.

8. Sharief MK, Hentges R. Association between tumor necrosis factor-alpha and disease progression in patients with multiple sclerosis. N Engl J Med. 1991;325(7):467-472.

9. The Lenercept Multiple Sclerosis Study Group The University of British Columbia MS/MRI Analysis Group. TNF neutralization in MS: results of a randomized, placebo-controlled multicenter study. Neurology. 1999;53(3):457-465.

10. Kaltsonoudis E, Voulgari PV, Konitsiotis S, Drosos AA. Demyelination and other neurological adverse events after anti-TNF therapy. Autoimmun Rev. 2014;13(1):54-58.

11. Eugster HP, Frei K, Bachmann R, Bluethmann H, Lassmann H, Fontana A. Severity of symptoms and demyelination in MOG-induced EAE depends on TNFR1. Eur J Immunol. 1999;29(2):626-632.

12. Suen WE, Bergman CM, Hjelmström P, Ruddle NH. A critical role for lymphotoxin in experimental allergic encephalomyelitis J Exp Med. 1997;186(8):1233-1240.

13. Tsakiri N, Papadopoulos D, Denis MC, Mitsikostas DD, Kollias G. TNFR2 on non-haematopoietic cells is required for Foxp3+ Treg-cell function and disease suppression in EAE. Eur J Immunol. 2012;42(2):403-412.

14. Gao H, et al. Opposing functions of microglial and macrophagic TNFR2 in the pathogenesis of experimental autoimmune encephalomyelitis. Cell Rep. 2017;18(1):198-212.

15. Arnett HA, Mason J, Marino M, Suzuki K, Matsushima GK, Ting JP. TNF alpha promotes proliferation of oligodendrocyte progenitors and remyelination. Nat Neurosci. 2001;4(11):1116-1122.

16. Patel JR, et al. Astrocyte TNFR2 is required for CXCL12-mediated regulation of oligodendrocyte progenitor proliferation and differentiation within the adult CNS. Acta Neuropathol. 2012;124(6):847-860.

17. Brambilla R, et al. Inhibition of soluble tumour necrosis factor is therapeutic in experimental autoimmune encephalomyelitis and promotes axon preservation and remyelination. Brain. 2011;134(Pt 9):2736-2754.

18. Lambertsen KL, et al. Microglia and macrophages express tumor necrosis factor receptor p75 following middle cerebral artery occlusion in mice. Neuroscience. 2007;144(3):934-949.

19. Madsen PM, et al. Oligodendroglial TNFR2 mediates membrane TNF-dependent repair in experimental autoimmune encephalomyelitis by promoting oligodendrocyte differentiation and remyelination. J Neurosci. 2016;36(18):5128-5143.

20. Fischer R, Wajant H, Kontermann R, Pfizenmaier K, Maier O. Astrocyte-specific activation of TNFR2 promotes oligodendrocyte maturation by secretion of leukemia inhibitory factor. Glia. 2014;62(2):272-283.

21. International Multiple Sclerosis Genetics Consortium, et al. Genetic risk and a primary role for cell-mediated immune mechanisms in multiple sclerosis. Nature. 2011;476(7359):214-219.

22. De Jager PL, et al. Meta-analysis of genome scans and replication identify CD6, IRF8 and TNFRSF1A as new multiple sclerosis susceptibility loci. Nat Genet. 2009;41(7):776-782.

23. Gregory AP, et al. TNF receptor 1 genetic risk mirrors outcome of anti-TNF therapy in multiple sclerosis. Nature. 2012;488(7412):508-511.

24. Wood H. Multiple sclerosis: TNF receptor 1 gene variant could explain failure of TNF-blocking drugs in multiple sclerosis. Nat Rev Neurol. 2012;8(9):476.

25. Martino G, et al. Tumor necrosis factor alpha and its receptors in relapsing-remitting multiple sclerosis. J Neurol Sci. 1997;152(1):51-61.

26. Suvannavejh GC, Lee HO, Padilla J, Dal Canto MC, Barrett TA, Miller SD. Divergent roles for p55 and p75 tumor necrosis factor receptors in the pathogenesis of MOG(35-55)-induced experimental autoimmune encephalomyelitis. Cell Immunol. 2000;205(1):24-33.

27. Probert L, et al. TNFR1 signalling is critical for the development of demyelination and the limitation of T-cell responses during immune-mediated CNS disease. Brain. 2000;123(Pt 10):2005-2019.

28. Schmidt H, Williamson D, Ashley-Koch A. HLA-DR15 haplotype and multiple sclerosis: a HuGE review. Am J Epidemiol. 2007;165(10):1097-1109.

29. Nalawade SA, Ji N, Raphael I, Pratt A, Kraig E, Forsthuber TG. Aire is not essential for regulating neuroinflammatory disease in mice transgenic for human autoimmune-diseases associated MHC class II genes HLA-DR2b and HLA-DR4. Cell Immunol. 2018;331:38-48.

30. Wilkins A. Cerebellar dysfunction in multiple sclerosis. Front Neurol. 2017;8:312.

31. Mehta AK, Gracias DT, Croft M. TNF activity and T cells. Cytokine. 2018;101:14-18.

32. Chen X, Wu X, Zhou Q, Howard OM, Netea MG, Oppenheim JJ. TNFR2 is critical for the stabilization of the CD4+Foxp3+ regulatory T. cell phenotype in the inflammatory environment. J Immunol. 2013;190(3):1076-1084.

33. Noack M, Miossec P. Th17 and regulatory T cell balance in autoimmune and inflammatory diseases. Autoimmun Rev. 2014;13(6):668-677. 
34. Zepp J, Wu L, Li X. IL-17 receptor signaling and T helper 17-mediated autoimmune demyelinating disease. Trends Immunol. 2011;32(5):232-239.

35. McCoy MK, Tansey MG. TNF signaling inhibition in the CNS: implications for normal brain function and neurodegenerative disease. J Neuroinflammation. 2008;5:45.

36. Meares GP, Ma X, Qin H, Benveniste EN. Regulation of CCL20 expression in astrocytes by IL-6 and IL-17. Glia. 2012;60(5):771-781.

37. Maysami S, Nguyen D, Zobel F, Heine S, Höpfner M, Stangel M. Oligodendrocyte precursor cells express a functional chemokine receptor CCR3: implications for myelination. J Neuroimmunol. 2006;178(1-2):17-23.

38. Lee YS, et al. Crosstalk between CCL7 and CCR3 promotes metastasis of colon cancer cells via ERK-JNK signaling pathways. Oncotarget. 2016;7(24):36842-36853

39. Mayo L, et al. Regulation of astrocyte activation by glycolipids drives chronic CNS inflammation. Nat Med. 2014;20(10):1147-1156.

40. Rostami A, Ciric B. Astrocyte-derived lactosylceramide implicated in multiple sclerosis. Nat Med. 2014;20(10):1092-1093.

41. Rothhammer V, et al. Sphingosine 1-phosphate receptor modulation suppresses pathogenic astrocyte activation and chronic progressive CNS inflammation. Proc Natl Acad Sci USA. 2017;114(8):2012-2017.

42. Kuno R, et al. The role of TNF-alpha and its receptors in the production of NGF and GDNF by astrocytes. Brain Res. 2006;1116(1):12-18.

43. Ambrosini E, Columba-Cabezas S, Serafini B, Muscella A, Aloisi F. Astrocytes are the major intracerebral source of macrophage inflammatory protein-3alpha/CCL20 in relapsing experimental autoimmune encephalomyelitis and in vitro. Glia. 2003;41(3):290-300.

44. Parajuli B, Horiuchi H, Mizuno T, Takeuchi H, Suzumura A. CCL11 enhances excitotoxic neuronal death by producing reactive oxygen species in microglia. Glia. 2015;63(12):2274-2284.

45. Jiang BC, et al. CXCL13 drives spinal astrocyte activation and neuropathic pain via CXCR5. J Clin Invest. 2016;126(2):745-761

46. Adzemovic MZ, et al. Expression of Cc111 associates with immune response modulation and protection against neuroinflammation in rats. PLoS One. 2012;7(7):e39794.

47. Ooi JD, et al. Dominant protection from HLA-linked autoimmunity by antigen-specific regulatory T cells. Nature. 2017;545(7653):243-247.

48. El-Tahan RR, Ghoneim AM, El-Mashad N. TNF- $\alpha$ gene polymorphisms and expression. Springerplus. 2016;5(1):1508.

49. Till A, et al. The Met-196 -\&gt; Arg variation of human tumor necrosis factor receptor 2 (TNFR2) affects TNF-alpha-induced apoptosis by impaired NF-kappaB signaling and target gene expression. J Biol Chem. 2005;280(7):5994-6004.

50. Song GG, Bae SC, Lee YH. Associations between functional TNFR2 $196 \mathrm{M} / \mathrm{R}$ polymorphisms and susceptibility to rheumatoid arthritis: a meta-analysis. Rheumatol Int. 2014;34(11):1529-1537.

51. Titelbaum DS, Degenhardt A, Kinkel RP. Anti-tumor necrosis factor alpha-associated multiple sclerosis. AJNR Am J Neuroradiol. $2005 ; 26(6): 1548-1550$.

52. Colombo E, Farina C. Astrocytes: key regulators of neuroinflammation. Trends Immunol. 2016;37(9):608-620

53. Correale J, Farez MF. The role of astrocytes in multiple sclerosis progression. Front Neurol. 2015;6:180.

54. Rothhammer V, Quintana FJ. Control of autoimmune CNS inflammation by astrocytes. Semin Immunopathol. 2015;37(6):625-638.

55. Nair A, Frederick TJ, Miller SD. Astrocytes in multiple sclerosis: a product of their environment. Cell Mol Life Sci. 2008;65(17):2702-2720.

56. Liddelow SA, Barres BA. Reactive astrocytes: production, function, and therapeutic potential. Immunity. 2017;46(6):957-967.

57. Zou J, et al. Glutamine synthetase down-regulation reduces astrocyte protection against glutamate excitotoxicity to neurons. Neurochem Int. 2010;56(4):577-584.

58. Selmaj KW, Farooq M, Norton WT, Raine CS, Brosnan CF. Proliferation of astrocytes in vitro in response to cytokines. A primary role for tumor necrosis factor. J Immunol. 1990;144(1):129-135.

59. Londoño AC, Mora CA. Role of CXCL13 in the formation of the meningeal tertiary lymphoid organ in multiple sclerosis. F1000Res. 2018;7:514.

60. International Multiple Sclerosis Genetics Consortium, et al. MANBA, CXCR5, SOX8, RPS6KB1 and ZBTB46 are genetic risk loci for multiple sclerosis. Brain. 2013;136(Pt 6):1778-1782.

61. Krumbholz M, et al. Chemokines in multiple sclerosis: CXCL12 and CXCL13 up-regulation is differentially linked to CNS immune cell recruitment. Brain. 2006;129(Pt 1):200-211.

62. Van Wagoner NJ, Oh JW, Repovic P, Benveniste EN. Interleukin-6 (IL-6) production by astrocytes: autocrine regulation by IL-6 and the soluble IL-6 receptor. J Neurosci. 1999;19(13):5236-5244.

63. Campbell IL, et al. Neurologic disease induced in transgenic mice by cerebral overexpression of interleukin 6. Proc Natl Acad Sci USA. 1993;90(21):10061-10065.

64. Van Wagoner NJ, Benveniste EN. Interleukin-6 expression and regulation in astrocytes. J Neuroimmunol. 1999;100(1-2):124-139.

65. Savarin C, et al. Astrocyte response to IFN- $\gamma$ limits IL-6-mediated microglia activation and progressive autoimmune encephalomyelitis. J Neuroinflammation. 2015;12:79.

66. Imitola J, et al. Elevated expression of granulocyte-macrophage colony-stimulating factor receptor in multiple sclerosis lesions. J Neuroimmunol. 2018;317:45-54.

67. Becher B, Tugues S, Greter M. GM-CSF: from growth factor to central mediator of tissue inflammation. Immunity. 2016;45(5):963-973.

68. Ludwin SK, Rao VTs, Moore CS, Antel JP. Astrocytes in multiple sclerosis. Mult Scler. 2016;22(9):1114-1124.

69. Chabas D, et al. The influence of the proinflammatory cytokine, osteopontin, on autoimmune demyelinating disease. Science. 2001;294(5547):1731-1735.

70. Domingues HS, Portugal CC, Socodato R, Relvas JB. Oligodendrocyte, astrocyte, and microglia crosstalk in myelin development, damage, and repair. Front Cell Dev Biol. 2016;4:71

71. Du F, et al. Inflammatory Th17 cells express integrin $\alpha v \beta 3$ for pathogenic function. Cell Rep. 2016;16(5):1339-1351.

72. Steinman L. A brief history of $\mathrm{T}(\mathrm{H}) 17$, the first major revision in the $\mathrm{T}(\mathrm{H}) 1 / \mathrm{T}(\mathrm{H}) 2$ hypothesis of $\mathrm{T}$ cell-mediated tissue damage. Nat Med. 2007;13(2):139-145. 
73. Miyamoto N, et al. Astrocytes promote oligodendrogenesis after white matter damage via brain-derived neurotrophic factor J Neurosci. 2015;35(41):14002-14008.

74. Kang Z, et al. Astrocyte-restricted ablation of interleukin-17-induced Act1-mediated signaling ameliorates autoimmune encephalomyelitis. Immunity. 2010;32(3):414-425.

75. Ito M, et al. Brain regulatory T cells suppress astrogliosis and potentiate neurological recovery. Nature. 2019;565(7738):246-250

76. Mayo L, et al. IL-10-dependent Tr1 cells attenuate astrocyte activation and ameliorate chronic central nervous system inflammation. Brain. 2016;139(Pt 7):1939-1957.

77. Mangalam A, et al. HLA-DQ8 (DQB1*0302)-restricted Th17 cells exacerbate experimental autoimmune encephalomyelitis in HLA-DR3-transgenic mice. J Immunol. 2009;182(8):5131-5139.

78. Shultz LD, et al. Generation of functional human T-cell subsets with HLA-restricted immune responses in HLA class I expressing NOD/SCID/IL2r gamma(null) humanized mice. Proc Natl Acad Sci USA. 2010;107(29):13022-13027.

79. Atretkhany KN, et al. Intrinsic TNFR2 signaling in T regulatory cells provides protection in CNS autoimmunity. Proc Natl Acad Sci USA. 2018;115(51):13051-13056.

80. Miller PG, Bonn MB, McKarns SC. Transmembrane TNF-TNFR2 impairs Th17 differentiation by promoting Il2 expression. J Immunol. 2015;195(6):2633-2647.

81. Rich C, et al. Myelin oligodendrocyte glycoprotein-35-55 peptide induces severe chronic experimental autoimmune encephalomyelitis in HLA-DR2-transgenic mice. Eur J Immunol. 2004;34(5):1251-1261.

82. Kawamura K, McLaughlin KA, Weissert R, Forsthuber TG. Myelin-reactive type B T cells and T cells specific for low-affinity MHC-binding myelin peptides escape tolerance in HLA-DR transgenic mice. J Immunol. 2008;181(5):3202-3211.

83. Erickson SL, et al. Decreased sensitivity to tumour-necrosis factor but normal T-cell development in TNF receptor-2-deficient mice. Nature. 1994;372(6506):560-563.

84. Madsen L, et al. Mice lacking all conventional MHC class II genes. Proc Natl Acad Sci USA. 1999;96(18):10338-10343.

85. Guyenet SJ, Furrer SA, Damian VM, Baughan TD, La Spada AR, Garden GA. A simple composite phenotype scoring system for evaluating mouse models of cerebellar ataxia. J Vis Exp. 2010;(39):1787.

86. McCloy RA, Rogers S, Caldon CE, Lorca T, Castro A, Burgess A. Partial inhibition of Cdk1 in G 2 phase overrides the SAC and decouples mitotic events. Cell Cycle. 2014;13(9):1400-1412.

87. Kim RY, et al. Astrocyte CCL2 sustains immune cell infiltration in chronic experimental autoimmune encephalomyelitis J Neuroimmunol. 2014;274(1-2):53-61.

88. Raphael I, et al. Serum neuroinflammatory disease-induced central nervous system proteins predict clinical onset of experimental autoimmune encephalomyelitis. Front Immunol. 2017;8:812. 